\title{
Rate Region of Gaussian Multiple Description Coding With Individual and Central Distortion Constraints
}

\author{
Jun Chen, Member, IEEE
}

\begin{abstract}
The rate region of Gaussian multiple description coding with individual and central distortion constraints is completely characterized. Specifically, a lower bound and an upper bound are derived for each supporting hyperplane of the rate region, where the lower bound is associated with a max-min game while the upper bound is associated with a min-max game; furthermore, it is shown that these two bounds coincide due to the existence of a saddle point.
\end{abstract}

Index Terms-Contra-polymatroid, entropy power inequality, min-max, multiple description coding, saddle point.

\section{INTRODUCTION}

$\mathbf{M}$ ULTIPLE description coding is a quantization technique developed for multimedia transmission through unreliable links. In the standard setting of the multiple description problem, several coded representations are formed for the target source such that any subset of these representations can be used to reconstruct the source with certain fidelity. Each representation is referred to as a description, and the goal is to find the optimal tradeoff between the code rates of the descriptions and the reconstruction distortions.

The first general result on the multiple description problem is the 2-description achievable rate region by El Gamal and Cover (EGC) [1], which is based on the idea of correlated quantization. This result has been extended to the general $L$-description case in [2]. It is observed in [3] and [4] that the EGC achievable rate region can be enlarged by coupling correlated quantization with the binning technique. Further improvements can be found in [5].

Although the general multiple description problem is still widely open, several conclusive results have been obtained for the quadratic Gaussian case. Notably, when only individual and central distortion constraints ${ }^{1}$ are imposed, the minimum achievable sum-rate has been derived by Ozarow for the 2-description case [6] and by Wang and Viswanath for the general $L$-description case [7] (see also [2] for the symmetric case). Moreover, the minimum achievable sum-rate together with two trivial bounds on the individual rates yields an exact characterization of the rate region for the 2-description case [6]. However,

Manuscript received April 30, 2008; revised April 21, 2009. Current version published August 19, 2009.

The author is with the Department of Electrical and Computer Engineering, McMaster University, Hamilton, ON L8S 4K1, Canada (e-mail: junchen@ece. mcmaster.ca).

Communicated by I. Kontoyiannis, Associate Editor for Shannon Theory.

Digital Object Identifier 10.1109/TIT.2009.2025540

${ }^{1}$ That is to say, the distortion constraints are only imposed on the individual descriptions and on the complete set of descriptions. the rate region of Gaussian multiple description coding with individual and central distortion constraints for the general $L$-description case is still unknown. The key contribution of the present work is a complete solution to this open problem.

Throughout this paper, the logarithm function is to base $e$. We use $I_{n}$ to denote an $n \times n$ identity matrix and use $A^{n}$ to denote $n$-dimensional random vector $(A(1), \ldots, A(n))$ for any positive integer $n$. The operators $\mathbb{E}(\cdot)$ and $\operatorname{tr}(\cdot)$ denote expectation and trace, respectively. For random vectors $A^{n}$ and $B^{m}$, the conditional expectation of $A^{n}$ given $B^{m}$ is denoted by $\mathbb{E}\left(A^{n} \mid B^{m}\right)$ and the covariance matrix of $A^{n}-\mathbb{E}\left(A^{n} \mid B^{m}\right)$ is denoted by $\operatorname{cov}\left(A^{n} \mid B^{m}\right)$; with a slight abuse of notation, we interpret $\operatorname{cov}\left(A^{n} \mid B^{m}\right)$ as $\operatorname{tr}\left(\operatorname{cov}\left(A^{n} \mid B^{m}\right)\right)$ when $n=1$. We use the convention that $\frac{1}{0}=\infty, \log 0=-\infty$, and $0 \log 0=0$.

\section{Problem Setting And Main Results}

Let $\{X(m)\}_{m=1}^{\infty}$ be a stationary and memoryless Gaussian process with mean zero and variance $\sigma_{X}^{2}$. Given individual distortion constraints $d_{\{i\}}, i=1, \ldots, L$, and central distortion constraint $d_{\{1, \ldots, L\}}$, we say a rate vector $\left(R_{1}, \ldots, R_{L}\right)$ is achievable if there exist, for all sufficiently large $n$, encoding functions $f_{i}^{(n)}: \mathcal{R}^{n} \rightarrow \mathcal{C}_{i}^{(n)}$ with $\frac{1}{n} \log \left|\mathcal{C}_{i}^{(n)}\right| \leq R_{i}$, $i=1, \ldots, L$, such that

$$
\begin{aligned}
& \frac{1}{n} \operatorname{tr}\left(\operatorname{cov}\left(X^{n} \mid f_{i}^{(n)}\left(X^{n}\right)\right)\right) \leq d_{\{i\}}, \quad i=1, \ldots, L, \\
& \frac{1}{n} \operatorname{tr}\left(\operatorname{cov}\left(X^{n} \mid f_{1}^{(n)}\left(X^{n}\right), \ldots, f_{L}^{(n)}\left(X^{n}\right)\right)\right) \leq d_{\{1, \ldots, L\}} .
\end{aligned}
$$

The rate region $\mathcal{R}\left(d_{\{1\}}, \ldots, d_{\{L\}}, d_{\{1, \ldots, L\}}\right)$ is the convex closure of the set of all achievable rate vectors with respect to individual distortion constraints $d_{\{i\}}, i=1, \ldots, L$, and central distortion constraint $d_{\{1, \ldots, L\}}$. Without loss of generality, we shall assume $0<d_{\{1\}}, \ldots, d_{\{L\}}, d_{\{1, \ldots, L\}} \leq \sigma_{X}^{2}$.

Since $\mathcal{R}\left(d_{\{1\}}, \ldots, d_{\{L\}}, d_{\{1, \ldots, L\}}\right)$ is a closed convex set, it suffices to characterize its supporting hyperplanes, which boils down to solving the following optimization problem:

$$
\min _{\left(R_{1}, \ldots, R_{L}\right) \in \mathcal{R}\left(d_{\{1\}}, \ldots, d_{\{L\}}, d_{\{1, \ldots, L\}}\right)} \sum_{i=1}^{L} \alpha_{i} R_{i}
$$

where $\alpha_{i}, i=1, \ldots, L$, are arbitrary nonnegative numbers. It is clear that through suitable relabeling, we may assume, with no loss of generality, that $\alpha_{1} \geq \cdots \geq \alpha_{L}$. Moreover, if $\alpha_{L}=0$, then we can allocate enough rate to description $L$ to meet the individual distortion constraint $d_{\{L\}}$ and the cental distortion constraint $d_{\{1, \ldots, L\}}$ without affecting the weighted sum-rate while 
the other descriptions only need to meet their corresponding individual distortion constraints; as a consequence, it follows from the classic rate-distortion theory that

$$
\begin{aligned}
\min _{\left(R_{1}, \ldots, R_{L}\right) \in \mathcal{R}\left(d_{\{1\}}, \ldots, d_{\{L\}}, d_{\{1, \ldots, L\}}\right)} \sum_{i=1}^{L} \alpha_{i} R_{i} \\
=\sum_{i=1}^{L-1} \frac{\alpha_{i}}{2} \log \left(\frac{\sigma_{X}^{2}}{d_{\{i\}}}\right) \\
=\sum_{i=1}^{L} \frac{\alpha_{i}}{2} \log \left(\frac{\sigma_{X}^{2}}{d_{\{i\}}}\right) .
\end{aligned}
$$

Therefore, we shall assume $\alpha_{1} \geq \cdots \geq \alpha_{L}>0$ in the rest of this paper.

Note that when $\alpha_{1}=\cdots=\alpha_{L}$, the optimization problem (1) yields the minimum sum-rate (up to a scalar) of the rate region $\mathcal{R}\left(d_{\{1\}}, \ldots, d_{\{L\}}, d_{\{1, \ldots, L\}}\right)$. Specifically, it is known [6], [7] that

$$
\begin{gathered}
\min _{\left(R_{1}, \ldots, R_{L}\right) \in \mathcal{R}\left(d_{\{1\}}, \ldots, d_{\{L\}}, d_{\{1, \ldots, L\}}\right)} \sum_{i=1}^{L} R_{i} \\
=\sup _{\sigma_{Z}^{2}>0} \frac{1}{2} \log \left(\frac{\sigma_{X}^{2}\left(\sigma_{X}^{2}+\sigma_{Z}^{2}\right)^{L-1}\left(d_{\{1, \ldots, L\}}+\sigma_{Z}^{2}\right)}{d_{\{1, \ldots, L\}} \prod_{i=1}^{L}\left(d_{\{i\}}+\sigma_{Z}^{2}\right)}\right) .
\end{gathered}
$$

However, the technique used to derive (2) is not sufficient for characterizing other supporting hyperplanes. The main reason is as follows. To minimize the achievable sum-rate of the EGC scheme, the covariance matrix of the quantization errors associated with different descriptions has the property that all the off-diagonal entries are identical and nonpositive. As shown by the ingenious converse argument devised by Ozarow [6] (see also [7]), this property can be effectively exploited to create a conditional independence structure by introducing a hidden auxiliary random vector. Remarkably, the resulting lower bound coincides with the minimum EGC achievable sum-rate. Unfortunately, when optimizing the EGC achievable scheme for other hyperplanes, the corresponding covariance matrix of the quantization errors in general does not have this simple structure. As a consequence, a direct application of Ozarow's argument does not lead to a matching lower bound, and more sophisticated techniques are needed.
To the end of solving the optimization problem (1), we shall introduce several auxiliary distortion constraints in addition to the individual and central distortion constraints. We say the weighted sum-rate $R$ is achievable with respect to individual distortion constraints $d_{\{i\}}, i=1, \ldots, L$, auxiliary distortion constraints $d_{\{1, \ldots, i\}}^{\prime}, i=2, \ldots, L-1$, and central distortion constraint $d_{\{1, \ldots, L\}}$, if there exist, for all sufficiently large $n$, encoding functions $f_{i}^{(n)}: \mathcal{R}^{n} \rightarrow \mathcal{C}_{i}^{(n)}, i=1, \ldots, L$, with $\frac{1}{n} \sum_{i=1}^{L} \alpha_{i} \log \left|\mathcal{C}_{i}^{(n)}\right| \leq R$ such that

$$
\begin{aligned}
& \frac{1}{n} \operatorname{tr}\left(\operatorname{cov}\left(X^{n} \mid f_{i}^{(n)}\left(X^{n}\right)\right)\right) \\
& \quad \leq d_{\{i\}}, \quad i=1, \ldots, L, \\
& \frac{1}{n} \operatorname{tr}\left(\operatorname{cov}\left(X^{n} \mid f_{1}^{(n)}\left(X^{n}\right), \ldots, f_{i}^{(n)}\left(X^{n}\right)\right)\right) \\
& \quad \leq d_{\{1, \ldots, i\}}^{\prime}, \quad i=2, \ldots, L-1, \\
& \frac{1}{n} \operatorname{tr}\left(\operatorname{cov}\left(X^{n} \mid f_{1}^{(n)}\left(X^{n}\right), \ldots, f_{L}^{(n)}\left(X^{n}\right)\right)\right) \\
& \quad \leq d_{\{1, \ldots, L\}} .
\end{aligned}
$$

Let $\kappa\left(d_{\{1\}}, \ldots, d_{\{L\}}, d_{\{1,2\}}^{\prime}, \ldots, d_{\{1, \ldots, L-1\}}^{\prime}, d_{\{1, \ldots, L\}}\right)$ be the infimum of all achievable weighted sum-rate $R$ with respect to individual distortion constraints $d_{\{i\}}, i=1, \ldots, L$, auxiliary distortion constraints $d_{\{1, \ldots, i\}}^{\prime}, i=2, \ldots, L-1$, and central distortion constraint $d_{\{1, \ldots, L\}}$. Without loss of generality, we shall assume $0<d_{\{1,2\}}^{\prime}, \ldots, d_{\{1, \ldots, L-1\}}^{\prime} \leq \sigma_{X}^{2}$. Note that the auxiliary distortion constraints are void if $L=2$.

We define $\psi(\cdot)$ shown at the bottom of the page. The importance of this function is evident from the following theorems.

Theorem 1: We have

$$
\begin{aligned}
& \kappa\left(d_{\{1\}}, \ldots, d_{\{L\}}, d_{\{1,2\}}^{\prime}, \ldots, d_{\{1, \ldots, L-1\}}^{\prime}, d_{\{1, \ldots, L\}}\right) \\
& \quad \max _{\sigma_{j}^{2} \in\left[0, \sigma_{X}^{2}\right], j=1, \ldots, L-1} d_{\{1, \ldots, i\}} \in\left[0, d_{\{1, \ldots, i\}}^{\prime}\right], i=2, \ldots, L-1 \\
& \quad \psi\left(d_{\{1\}}, \ldots, d_{\{L\}}, d_{\{1,2\}}, \ldots, d_{\{1, \ldots, L\}}, \sigma_{1}^{2}, \ldots, \sigma_{L-1}^{2}\right) .
\end{aligned}
$$

Therefore, $\kappa\left(d_{\{1\}}, \ldots, d_{\{L\}}, d_{\{1,2\}}^{\prime}, \ldots, d_{\{1, \ldots, L-1\}}^{\prime}, d_{\{1, \ldots, L\}}\right)$ can be lower-bounded using a max-min game. Interestingly, the corresponding min-max game yields an upper bound on $\kappa\left(d_{\{1\}}, \ldots, d_{\{L\}}, d_{\{1,2\}}^{\prime}, \ldots, d_{\{1, \ldots, L-1\}}^{\prime}, d_{\{1, \ldots, L\}}\right)$.

Theorem 2: We have (4) shown at the bottom of the page.

$$
\begin{aligned}
\psi( & \left.d_{\{1\}}, \ldots, d_{\{L\}}, d_{\{1,2\}}, \ldots, d_{\{1, \ldots, L\}}, \sigma_{1}^{2}, \ldots, \sigma_{L-1}^{2}\right) \\
= & \sum_{i=1}^{L-1}\left(\frac{\alpha_{i+1}}{2} \log \left(\frac{\sigma_{X}^{4}\left(\sigma_{X}^{2} d_{\{1, \ldots, i+1\}}-d_{\{1, \ldots, i+1\}} \sigma_{i}^{2}+\sigma_{X}^{2} \sigma_{i}^{2}\right)}{\left(\sigma_{X}^{2} d_{\{1, \ldots, i\}}-d_{\{1, \ldots, i\}} \sigma_{i}^{2}+\sigma_{X}^{2} \sigma_{i}^{2}\right)\left(\sigma_{X}^{2} d_{\{i+1\}}-d_{\{i+1\}} \sigma_{i}^{2}+\sigma_{X}^{2} \sigma_{i}^{2}\right)}\right)\right. \\
& \left.+\frac{\alpha_{i}-\alpha_{i+1}}{2} \log \left(\frac{\sigma_{X}^{2}}{d_{\{1, \ldots, i\}}}\right)\right)+\frac{\alpha_{L}}{2} \log \left(\frac{\sigma_{X}^{2}}{d_{\{1, \ldots, L\}}}\right) .
\end{aligned}
$$

$$
\begin{aligned}
& \kappa\left(d_{\{1\}}, \ldots, d_{\{L\}}, d_{\{1,2\}}^{\prime}, \ldots, d_{\{1, \ldots, L-1\}}^{\prime}, d_{\{1, \ldots, L\}}\right) \\
& \quad \leq \min _{d_{\{1, \ldots, i\}} \in\left[0, d_{\{1, \ldots, i\}}^{\prime}\right], i=2, \ldots, L-1 \sigma_{j}^{2} \in\left[0, \sigma_{X}^{2}\right], j=1, \ldots, L-1} \psi\left(d_{\{1\}}, \ldots, d_{\{L\}}, d_{\{1,2\}}, \ldots, d_{\{1, \ldots, L\}}, \sigma_{1}^{2}, \ldots, \sigma_{L-1}^{2}\right)
\end{aligned}
$$


It turns out that max-min (cf. (3)) and min-max (cf. (4)) coincide, yielding a complete characterization of $\kappa\left(d_{\{1\}}, \ldots, d_{\{L\}}, d_{\{1,2\}}^{\prime}, \ldots, d_{\{1, \ldots, L-1\}}^{\prime}, d_{\{1, \ldots, L\}}\right)$.

Theorem 3: We have the first set of equations shown at the bottom of the following page.

One can immediately obtain the following result by setting $d_{\{1, \ldots, i\}}^{\prime}=\sigma_{X}^{2}, i=2, \ldots, L-1$, to make the auxiliary distortion constraints redundant.

Theorem 4: We have the second set of equations shown at the bottom of the page.

As a side note, the rate region $\mathcal{R}\left(d_{\{1\}}, \ldots, d_{\{L\}}, d_{\{1, \ldots, L\}}\right)$ is achievable using the lattice quantization scheme (possibly with timesharing) developed in [8] and [9].

It will be seen that besides Ozarow's method of creating conditional independence structure, several new ingredients are needed to prove these theorems, which include

- an application of Costa's entropy power inequality [10],

- multiple auxiliary random vectors,

- saddle point analysis of a max-min/min-max game,

- a delicate construction of the covariance matrix of the quantization errors for the EGC achievable scheme.

The remainder of this paper is divided into four sections. The proofs of Theorem 1, Theorem 2, and Theorem 3 are given in Sections III, IV, and V, respectively. We conclude the paper in Section VI.

\section{LOWER Bound: ProOf OF TheOREM 1}

The following lemma is of crucial importance for establishing the desired lower bound.

Lemma 1: Let $N_{1}^{n}$ and $N_{2}^{n}$ be two $n$-dimensional Gaussian random vectors with positive definite covariance matrices $\sigma_{1}^{2} I_{n}$ and $\sigma_{2}^{2} I_{n}$, respectively. Let $\mu_{2} \geq \mu_{1} \geq 0$ be two real numbers. For any $n$-dimensional random vector $S^{n}$ and random variable $T$, both independent of $N_{1}^{n}$ and $N_{2}^{n}$, such that $\operatorname{tr}\left(\operatorname{cov}\left(S^{n} \mid T\right)\right) \leq n d^{\prime}$, we have

$$
\begin{aligned}
& \mu_{1} h\left(S^{n}+N_{1}^{n} \mid T\right)-\mu_{2} h\left(S^{n}+N_{2}^{n} \mid T\right) \\
& \quad+\left(\mu_{2}-\mu_{1}\right) h\left(S^{n} \mid T\right)
\end{aligned}
$$

$$
\begin{aligned}
\leq & \max _{d \in\left[0, d^{\prime}\right]} \frac{\mu_{1} n}{2} \log \left(d+\sigma_{1}^{2}\right) \\
& -\frac{\mu_{2} n}{2} \log \left(d+\sigma_{2}^{2}\right)+\frac{\left(\mu_{2}-\mu_{1}\right) n}{2} \log (d)
\end{aligned}
$$

where $h(\cdot)$ is the differential entropy function.

Remark: It is worth noting that the maximum in (5) is not necessarily attained at $d=d^{\prime}$.

Proof: See Appendix A.

Now we proceed to derive a lower bound on $\kappa\left(d_{\{1\}}, \ldots, d_{\{L\}}, d_{\{1,2\}}^{\prime}, \ldots, d_{\{1, \ldots, L-1\}}^{\prime}, d_{\{1, \ldots, L\}}\right)$. Let $f_{i}^{(n)}:$ $\mathcal{R}^{n} \rightarrow \mathcal{C}_{i}^{(n)}, i=1, \ldots, L$, be $L$ arbitrary encoding functions satisfying

$$
\begin{aligned}
& \frac{1}{n} \operatorname{tr}\left(\operatorname{cov}\left(X^{n} \mid f_{i}^{(n)}\left(X^{n}\right)\right)\right) \\
& \quad \leq d_{\{i\}}, \quad i=1, \ldots, L, \\
& \frac{1}{n} \operatorname{tr}\left(\operatorname{cov}\left(X^{n} \mid f_{1}^{(n)}\left(X^{n}\right), \ldots, f_{i}^{(n)}\left(X^{n}\right)\right)\right) \\
& \quad \leq d_{\{1, \ldots, i\}}^{\prime}, \quad i=2, \ldots, L-1, \\
& \frac{1}{n} \operatorname{tr}\left(\operatorname{cov}\left(X^{n} \mid f_{1}^{(n)}\left(X^{n}\right), \ldots, f_{L}^{(n)}\left(X^{n}\right)\right)\right) \\
& \quad \leq d_{\{1, \ldots, L\} .}
\end{aligned}
$$

Define $C_{i}=f_{i}^{(n)}\left(X^{n}\right), i=1, \ldots, L$. Note that

$$
\begin{aligned}
\sum_{i=1}^{L} \alpha_{i} \log \left|\mathcal{C}_{i}^{(n)}\right| & \geq \sum_{i=1}^{L} \alpha_{i} H\left(C_{i}\right) \\
& =\sum_{i=1}^{L}\left(\alpha_{i}-\alpha_{i+1}\right) \sum_{j=1}^{i} H\left(C_{j}\right)
\end{aligned}
$$

where $\alpha_{L+1} \triangleq 0$. It is easy to verify that

$$
\begin{aligned}
& \sum_{j=1}^{i} H\left(C_{j}\right) \\
& \quad=H\left(C_{1}, \ldots, C_{i}\right)+\sum_{j=1}^{i-1} I\left(C_{1}, \ldots, C_{j} ; C_{j+1}\right) \\
& =I\left(X^{n} ; C_{1}, \ldots, C_{i}\right)+\sum_{j=1}^{i-1} I\left(C_{1}, \ldots, C_{j} ; C_{j+1}\right), \\
& i=2, \ldots, L .
\end{aligned}
$$

$$
\begin{aligned}
& \kappa\left(d_{\{1\}}, \ldots, d_{\{L\}}, d_{\{1,2\}}^{\prime}, \ldots, d_{\{1, \ldots, L-1\}}^{\prime}, d_{\{1, \ldots, L\}}\right) \\
&=\max _{\sigma_{j}^{2} \in\left[0, \sigma_{X}^{2}\right], j=1, \ldots, L-1} d_{\{1, \ldots, i\}} \in\left[0, d_{\{1, \ldots, i\}}^{\prime}\right], i=2, \ldots, L-1 \\
&=\min _{d_{\{1, \ldots, i\}} \in\left[0, d_{\{1, \ldots, i\}}^{\prime}\right], i=2, \ldots, L-1} \psi\left(d_{\{1\}}, \ldots, d_{\{L\}}^{2} \in\left[0, \sigma_{X}^{2}\right], j=1, \ldots, L-1\right. \\
&\left.\min _{\{1,2\}}, \ldots, d_{\{1, \ldots, L\}}, \sigma_{1}^{2}, \ldots, \sigma_{L-1}^{2}\right)
\end{aligned}
$$

$$
\begin{aligned}
& \min _{\left(R_{1}, \ldots, R_{L}\right) \in \mathcal{R}\left(d_{\{1\}}, \ldots, d_{\{L\}}, d_{\{1, \ldots, L\}}\right)} \sum_{i=1}^{L} \alpha_{i} R_{i} \\
& =\max _{\sigma_{j}^{2} \in\left[0, \sigma_{X}^{2}\right], j=1, \ldots, L-1} \min _{d_{\{1, \ldots, i\}} \in\left[0, \sigma_{X}^{2}\right], i=2, \ldots, L-1} \psi\left(d_{\{1\}}, \ldots, d_{\{L\}}, d_{\{1,2\}}, \ldots, d_{\{1, \ldots, L\}}, \sigma_{1}^{2}, \ldots, \sigma_{L-1}^{2}\right) \\
& =\min _{d_{\{1, \ldots, i\}} \in\left[0, \sigma_{X}^{2}\right], i=2, \ldots, L-1} \max _{\sigma_{j}^{2} \in\left[0, \sigma_{X}^{2}\right], j=1, \ldots, L-1} \psi\left(d_{\{1\}}, \ldots, d_{\{L\}}, d_{\{1,2\}}, \ldots, d_{\{1, \ldots, L\}}, \sigma_{1}^{2}, \ldots, \sigma_{L-1}^{2}\right) .
\end{aligned}
$$


Substituting (7) into (6), we obtain

$$
\begin{aligned}
& \sum_{i=1}^{L} \alpha_{i} \log \left|\mathcal{C}_{i}^{(n)}\right| \\
& \geq \sum_{i=1}^{L}\left(\alpha_{i}-\alpha_{i+1}\right) I\left(X^{n} ; C_{1}, \ldots, C_{i}\right) \\
& \quad+\sum_{i=2}^{L}\left(\alpha_{i}-\alpha_{i+1}\right) \sum_{j=1}^{i-1} I\left(C_{1}, \ldots, C_{j} ; C_{j+1}\right) \\
& =\sum_{i=1}^{L}\left(\alpha_{i}-\alpha_{i+1}\right) I\left(X^{n} ; C_{1}, \ldots, C_{i}\right) \\
& \quad+\sum_{i=2}^{L} \alpha_{i} I\left(C_{1}, \ldots, C_{i-1} ; C_{i}\right) .
\end{aligned}
$$

Let $Z_{1}^{n}, \ldots, Z_{L-1}^{n}$ be zero-mean $n$-dimensional Gaussian random vectors with covariance matrices $\sigma_{Z_{1}}^{2} I_{n}, \ldots, \sigma_{Z_{L-1}}^{2} I_{n}$, respectively. We have

$$
\begin{aligned}
I\left(C_{1}, \ldots, C_{i-1} ; C_{i}\right) \\
= \\
\quad I\left(X^{n}+Z_{i-1}^{n} ; C_{1}, \ldots, C_{i-1}\right)+I\left(X^{n}+Z_{i-1}^{n} ; C_{i}\right) \\
\quad+I\left(C_{1}, \ldots, C_{i-1} ; C_{i} \mid X^{n}+Z_{i-1}^{n}\right) \\
\quad-I\left(X^{n}+Z_{i-1}^{n} ; C_{1}, \ldots, C_{i}\right) \\
\geq \\
\geq\left(X^{n}+Z_{i-1}^{n} ; C_{1}, \ldots, C_{i-1}\right) \\
\quad+I\left(X^{n}+Z_{i-1}^{n} ; C_{i}\right) \\
\quad-I\left(X^{n}+Z_{i-1}^{n} ; C_{1}, \ldots, C_{i}\right)
\end{aligned}
$$

which, together with (8), yields

$$
\begin{aligned}
& \sum_{i=1}^{L} \alpha_{i} \log \left|\mathcal{C}_{i}^{(n)}\right| \\
& \geq \sum_{i=1}^{L}\left(\alpha_{i}-\alpha_{i+1}\right) I\left(X^{n} ; C_{1}, \ldots, C_{i}\right) \\
& \quad+\sum_{i=2}^{L} \alpha_{i}\left(I\left(X^{n}+Z_{i-1}^{n} ; C_{1}, \ldots, C_{i-1}\right)\right. \\
& \quad+I\left(X^{n}+Z_{i-1}^{n} ; C_{i}\right) \\
&\left.\quad-I\left(X^{n}+Z_{i-1}^{n} ; C_{1}, \ldots, C_{i}\right)\right) \\
&=\left(\alpha_{1}-\alpha_{2}\right) I\left(X^{n} ; C_{1}\right)+\alpha_{2} I\left(X^{n}+Z_{1}^{n} ; C_{1}\right) \\
& \quad+\sum_{i=1}^{L-1} \alpha_{i+1} I\left(X^{n}+Z_{i}^{n} ; C_{i+1}\right) \\
& \quad+\sum_{i=2}^{L-1}\left(\alpha_{i+1} I\left(X^{n}+Z_{i}^{n} ; C_{1}, \ldots, C_{i}\right)\right. \\
& \quad-\alpha_{i} I\left(X^{n}+Z_{i-1}^{n} ; C_{1}, \ldots, C_{i}\right) \\
&\left.+\left(\alpha_{i}-\alpha_{i+1}\right) I\left(X^{n} ; C_{1}, \ldots, C_{i}\right)\right) \\
&+ \alpha_{L} I\left(X^{n}+Z_{L-1}^{n} ; C_{1}, \ldots, C_{L}\right) \\
&+\alpha_{L} I\left(X^{n} ; C_{1}, \ldots, C_{L}\right) .
\end{aligned}
$$

Note that

$$
\operatorname{tr}\left(\operatorname{cov}\left(X^{n} \mid C_{1}\right)\right) \leq n d_{\{1\}}
$$

$$
\begin{aligned}
& \operatorname{tr}\left(\operatorname{cov}\left(X^{n}+Z_{1}^{n} \mid C_{1}\right)\right) \\
& \quad=\operatorname{tr}\left(\operatorname{cov}\left(X^{n} \mid C_{1}\right)\right)+n \sigma_{Z_{1}}^{2} \leq n\left(d_{\{1\}}+\sigma_{Z_{1}}^{2}\right) \\
& \operatorname{tr}\left(\operatorname{cov}\left(X^{n}+Z_{i}^{n} \mid C_{i+1}\right)\right) \\
& \quad=\operatorname{tr}\left(\operatorname{cov}\left(X^{n} \mid C_{i+1}\right)\right)+n \sigma_{Z_{i}}^{2} \\
& \quad \leq n\left(d_{\{i+1\}}+\sigma_{Z_{i}}^{2}\right), \quad i=1, \ldots, L-1 .
\end{aligned}
$$

By the rate-distortion theorem

$$
\begin{aligned}
& I\left(X^{n} ; C_{1}\right) \geq \frac{n}{2} \log \left(\frac{\sigma_{X}^{2}}{d_{\{1\}}}\right), \\
& I\left(X^{n}+Z_{1}^{n} ; C_{1}\right) \geq \frac{n}{2} \log \left(\frac{\sigma_{X}^{2}+\sigma_{Z_{1}}^{2}}{d_{\{1\}}+\sigma_{Z_{1}}^{2}}\right), \\
& I\left(X^{n}+Z_{i}^{n} ; C_{i+1}\right) \geq \frac{n}{2} \log \left(\frac{\sigma_{X}^{2}+\sigma_{Z_{i}}^{2}}{d_{\{i+1\}}+\sigma_{Z_{i}}^{2}}\right),
\end{aligned}
$$

Moreover, we have

$$
\begin{aligned}
& \alpha_{i+1} I\left(X^{n}+Z_{i}^{n} ; C_{1}, \ldots, C_{i}\right) \\
&-\alpha_{i} I\left(X^{n}+Z_{i-1}^{n} ; C_{1}, \ldots, C_{i}\right) \\
&+\left(\alpha_{i}-\alpha_{i+1}\right) I\left(X^{n} ; C_{1}, \ldots, C_{i}\right) \\
&=\alpha_{i+1} h\left(X^{n}+Z_{i}^{n}\right)-\alpha_{i} h\left(X^{n}+Z_{i-1}^{n}\right) \\
& \quad+\left(\alpha_{i}-\alpha_{i+1}\right) h\left(X^{n}\right) \\
& \quad-\alpha_{i+1} h\left(X^{n}+Z_{i}^{n} \mid C_{1}, \ldots, C_{i}\right) \\
& \quad+\alpha_{i} h\left(X^{n}+Z_{i-1}^{n} \mid C_{1}, \ldots, C_{i}\right) \\
& \quad-\left(\alpha_{i}-\alpha_{i+1}\right) h\left(X^{n} \mid C_{1}, \ldots, C_{i}\right) \\
&=\frac{\alpha_{i+1} n}{2} \log \left(\sigma_{X}^{2}+\sigma_{Z_{i}}^{2}\right) \\
& \quad-\frac{\alpha_{i} n}{2} \log \left(\sigma_{X}^{2}+\sigma_{Z_{i-1}}^{2}\right) \\
& \quad+\frac{\left(\alpha_{i}-\alpha_{i+1}\right) n}{2} \log \left(\sigma_{X}^{2}\right) \\
& \quad-\alpha_{i+1} h\left(X^{n}+Z_{i}^{n} \mid C_{1}, \ldots, C_{i}\right) \\
& \quad+\alpha_{i} h\left(X^{n}+Z_{i-1}^{n} \mid C_{1}, \ldots, C_{i}\right) \\
& \quad-\left(\alpha_{i}-\alpha_{i+1}\right) h\left(X^{n} \mid C_{1}, \ldots, C_{i}\right), \\
& i=2, \ldots, L-1 .
\end{aligned}
$$

Since $\operatorname{tr}\left(\operatorname{cov}\left(X^{n} \mid C_{1}, \ldots, C_{i}\right)\right) \leq n d_{\{1, \ldots, i\}}^{\prime}$, it follows from Lemma 1 that

$$
\begin{aligned}
& \alpha_{i+1} h\left(X^{n}+Z_{i}^{n} \mid C_{1}, \ldots, C_{i}\right) \\
& -\alpha_{i} h\left(X^{n}+Z_{i-1}^{n} \mid C_{1}, \ldots, C_{i}\right) \\
& +\left(\alpha_{i}-\alpha_{i+1}\right) h\left(X^{n} \mid C_{1}, \ldots, C_{i}\right) \\
& \leq \max _{d_{\{1, \ldots, i\}} \in\left[0, d_{\{1, \ldots, i\}}^{\prime}\right]} \frac{\alpha_{i+1} n}{2} \log \left(d_{\{1, \ldots, i\}}+\sigma_{Z_{i}}^{2}\right) \\
& \quad-\frac{\alpha_{i} n}{2} \log \left(d_{\{1, \ldots, i\}}+\sigma_{Z_{i-1}}^{2}\right) \\
& +\frac{\left(\alpha_{i}-\alpha_{i+1}\right) n}{2} \log \left(d_{\{1, \ldots, i\}}\right) .
\end{aligned}
$$

Plugging (14) into (13), we obtain

$$
\begin{aligned}
& \alpha_{i+1} I\left(X^{n}+Z_{i}^{n} ; C_{1}, \ldots, C_{i}\right) \\
& \quad-\alpha_{i} I\left(X^{n}+Z_{i-1}^{n} ; C_{1}, \ldots, C_{i}\right) \\
& \quad+\left(\alpha_{i}-\alpha_{i+1}\right) I\left(X^{n} ; C_{1}, \ldots, C_{i}\right)
\end{aligned}
$$




$$
\begin{gathered}
\geq \min _{d_{\{1, \ldots, i\}} \in\left[0, d_{\{1, \ldots, i\}}^{\prime}\right]} \frac{\alpha_{i} n}{2} \log \left(\frac{d_{\{1, \ldots, i\}}+\sigma_{Z_{i-1}}^{2}}{\sigma_{X}^{2}+\sigma_{Z_{i-1}}^{2}}\right) \\
+\frac{\alpha_{i+1} n}{2} \log \left(\frac{\sigma_{X}^{2}+\sigma_{Z_{i}}^{2}}{d_{\{1, \ldots, i\}}+\sigma_{Z_{i}}^{2}}\right) \\
+\frac{\left(\alpha_{i}-\alpha_{i+1}\right) n}{2} \log \left(\frac{\sigma_{X}^{2}}{d_{\{1, \ldots, i\}}}\right), \\
i=2, \ldots, L-1 .
\end{gathered}
$$

Similarly, since $\operatorname{tr}\left(\operatorname{cov}\left(X^{n} \mid C_{1}, \ldots, C_{L}\right)\right) \leq n d_{\{1, \ldots, L\}}$, it follows from Lemma 1 that

$$
\begin{aligned}
& -\alpha_{L} h\left(X^{n}+Z_{L-1}^{n} \mid C_{1}, \ldots, C_{L}\right)+\alpha_{L} h\left(X^{n} \mid C_{1}, \ldots, C_{L}\right) \\
& \quad \leq \max _{d \in\left[0, d_{\{1, \ldots, L\}}\right]}-\frac{\alpha_{L} n}{2} \log \left(\frac{d+\sigma_{Z_{L-1}}^{2}}{d}\right) \\
& =-\frac{\alpha_{L} n}{2} \log \left(\frac{d_{\{1, \ldots, L\}}+\sigma_{Z_{L-1}}^{2}}{d_{\{1, \ldots, L\}}}\right) .
\end{aligned}
$$

Therefore, we have

$$
\begin{array}{rl}
-\alpha_{L} I & \left(X^{n}+Z_{L-1}^{n} ; C_{1}, \ldots, C_{L}\right) \\
+\alpha_{L} & I\left(X^{n} ; C_{1}, \ldots, C_{L}\right) \\
= & -\alpha_{L} h\left(X^{n}+Z_{L-1}^{n}\right) \\
& +\alpha_{L} h\left(X^{n}\right)+\alpha_{L} h\left(X^{n}+Z_{L-1}^{n} \mid C_{1}, \ldots, C_{L}\right) \\
& -\alpha_{L} h\left(X^{n} \mid C_{1}, \ldots, C_{L}\right) \\
\geq & \frac{\alpha_{L} n}{2} \log \left(\frac{\sigma_{X}^{2}}{\sigma_{X}^{2}+\sigma_{Z_{L-1}}^{2}}\right) \\
& +\frac{\alpha_{L} n}{2} \log \left(\frac{d_{\{1, \ldots, L\}}+\sigma_{Z_{L-1}}^{2}}{d_{\{1, \ldots, L\}}}\right) \\
= & \frac{\alpha_{L} n}{2} \log \left(\frac{\sigma_{X}^{2}\left(d_{\{1, \ldots, L\}}+\sigma_{Z_{L-1}}^{2}\right)}{d_{\{1, \ldots, L\}}\left(\sigma_{X}^{2}+\sigma_{Z_{L-1}}^{2}\right)}\right)
\end{array}
$$

Substituting (10), (11), (12), (15), and (16) into (9), we get

$$
\begin{aligned}
& \frac{1}{n} \sum_{i=1}^{L} \alpha_{i} \log \left|\mathcal{C}_{i}^{(n)}\right| \\
& \quad \underbrace{}_{d_{\{1, \ldots, i\}} \in\left[0, d_{\{1, \ldots, i\}}^{\prime}\right], i=2, \ldots, L-1} \phi\left(d_{\{1\}}, \ldots, d_{\{L\}}, d_{\{1,2\}}, \ldots,\right. \\
& \left.\quad d_{\{1, \ldots, L\}}, \sigma_{Z_{1}}^{2}, \ldots, \sigma_{Z_{L-1}}^{2}\right),
\end{aligned}
$$

where

$$
\begin{aligned}
\phi & \left(d_{\{1\}}, \ldots, d_{\{L\}}, d_{\{1,2\}}, \ldots, d_{\{1, \ldots, L\}}, \sigma_{Z_{1}}^{2}, \ldots, \sigma_{Z_{L-1}}^{2}\right) \\
= & \sum_{i=1}^{L-1}\left(\frac{\alpha_{i}-\alpha_{i+1}}{2} \log \left(\frac{\sigma_{X}^{2}}{d_{\{1, \ldots, i\}}}\right)\right. \\
& \left.+\frac{\alpha_{i+1}}{2} \log \left(\frac{\left(\sigma_{X}^{2}+\sigma_{Z_{i}}^{2}\right)\left(d_{\{1, \ldots, i+1\}}+\sigma_{Z_{i}}^{2}\right)}{\left(d_{\{1, \ldots, i\}}+\sigma_{Z_{i}}^{2}\right)\left(d_{\{i+1\}}+\sigma_{Z_{i}}^{2}\right)}\right)\right) \\
& +\frac{\alpha_{L}}{2} \log \left(\frac{\sigma_{X}^{2}}{d_{\{1, \ldots, L\}}}\right) .
\end{aligned}
$$

Since $\sigma_{Z_{j}}^{2}, j=1, \ldots, L-1$, can be arbitrary positive numbers, it follows that

$$
\begin{aligned}
& \frac{1}{n} \sum_{i=1}^{L} \alpha_{i} \log \left|\mathcal{C}_{i}^{(n)}\right| \\
& \quad \geq \sup _{\sigma_{Z_{j}}^{2}>0, j=1, \ldots, L-1} d_{\{1, \ldots, i\}} \in\left[0, d_{\{1, \ldots, i\}}^{\prime}\right], i=2, \ldots, L-1 \\
& \quad \phi\left(d_{\{1\}}, \ldots, d_{\{L\}}, d_{\{1,2\}}, \ldots, d_{\{1, \ldots, L\}}, \sigma_{Z_{1}}^{2}, \ldots, \sigma_{Z_{L-1}}^{2}\right) .
\end{aligned}
$$

Define a new function $\psi(\cdot)$ via the following equation:

$$
\begin{aligned}
& \psi\left(d_{\{1\}}, \ldots, d_{\{L\}}, d_{\{1,2\}}, \ldots, d_{\{1, \ldots, L\}}, \sigma_{1}^{2}, \ldots, \sigma_{L-1}^{2}\right) \\
& \quad=\phi\left(d_{\{1\}}, \ldots, d_{\{L\}}, d_{\{1,2\}}, \ldots, d_{\{1, \ldots, L\}}, \sigma_{Z_{1}}^{2}, \ldots, \sigma_{Z_{L-1}}^{2}\right)
\end{aligned}
$$

where $\sigma_{j}^{2}=\frac{\sigma_{X}^{2} \sigma_{Z_{j}}^{2}}{\sigma_{X}^{2}+\sigma_{Z_{j}}^{2}}, j=1, \ldots, L-1$. In view of the fact that there exists a one-to-one map between $\sigma_{Z_{i}}^{2} \in(0, \infty)$ and $\sigma_{i}^{2} \in\left(0, \sigma_{X}^{2}\right)$, we have

$$
\begin{aligned}
& \frac{1}{n} \sum_{i=1}^{L} \alpha_{i} \log \left|\mathcal{C}_{i}^{(n)}\right| \\
& \quad \geq \sup _{\sigma_{j}^{2} \in\left(0, \sigma_{X}^{2}\right), j=1, \ldots, L-1} d_{\{1, \ldots, i\}} \in\left[0, d_{\{1, \ldots, i\}}^{\prime}\right], i=2, \ldots, L-1 \\
& \quad \psi\left(d_{\{1\}}, \ldots, d_{\{L\}}, d_{\{1,2\}}, \ldots, d_{\{1, \ldots, L\}}, \sigma_{1}^{2}, \ldots, \sigma_{L-1}^{2}\right) .
\end{aligned}
$$

Note that the right-hand side of the above inequality is not affected if we extend the domain of $\sigma_{i}^{2}$ from $\left(0, \sigma_{X}^{2}\right)$ to $\left[0, \sigma_{X}^{2}\right]$. The proof is complete.

\section{UPPER BOUND: PROOF OF THEOREM 2}

We shall show that for any $\left(d_{\{1,2\}}, \ldots, d_{\{1, \ldots, L-1\}}\right)$ with $d_{\{1, \ldots, i\}} \in\left[0, d_{\{1, \ldots, i\}}^{\prime}\right], i=2, \ldots, L-1$,

$$
\begin{aligned}
& \kappa\left(d_{\{1\}}, \ldots, d_{\{L\}}, d_{\{1,2\}}^{\prime}, \ldots, d_{\{1, \ldots, L-1\}}^{\prime}, d_{\{1, \ldots, L\}}\right) \\
& \quad \leq \max _{\sigma_{j}^{2} \in\left[0, \sigma_{X}^{2}\right], j=1, \ldots, L-1} \\
& \quad \psi\left(d_{\{1\}}, \ldots, d_{\{L\}}, d_{\{1,2\}}, \ldots, d_{\{1, \ldots, L\}}, \sigma_{1}^{2}, \ldots, \sigma_{L-1}^{2}\right)
\end{aligned}
$$

from which Theorem 2 follows immediately. We assume $d_{\{1, \ldots, i\}}>0$ for all $i=2, \ldots, L-1$ since otherwise the right-hand side of the inequality is equal to infinity and (17) is trivially true.

Note that the maximization problem

$$
\begin{array}{r}
\max _{\sigma_{j}^{2} \in\left[0, \sigma_{X}^{2}\right], j=1, \ldots, L-1} \psi\left(d_{\{1\}}, \ldots, d_{\{L\}}, d_{\{1,2\}}, \ldots,\right. \\
\left.\quad d_{\{1, \ldots, L\}}, \sigma_{1}^{2}, \ldots, \sigma_{L-1}^{2}\right)
\end{array}
$$

can be decomposed into (18) shown at the top of the following page.

\section{Lemma 2: Define}

$$
\begin{aligned}
& d_{\{1, \ldots, j+1\}}^{\diamond}=\left(\frac{1}{d_{\{1, \ldots, j\}}}+\frac{1}{d_{\{j+1\}}}-\frac{1}{\sigma_{X}^{2}}\right)^{-1}, \\
& d_{\{1, \ldots, j+1\}}^{\star}=d_{\{1, \ldots, j\}}+d_{\{j+1\}}-\sigma_{X}^{2} .
\end{aligned}
$$




$$
\max _{\sigma_{j}^{2} \in\left[0, \sigma_{X}^{2}\right]} \log \left(\frac{\sigma_{X}^{4}\left(\sigma_{X}^{2} d_{\{1, \ldots, j+1\}}-d_{\{1, \ldots, j+1\}} \sigma_{j}^{2}+\sigma_{X}^{2} \sigma_{j}^{2}\right)}{\left(\sigma_{X}^{2} d_{\{1, \ldots, j\}}-d_{\{1, \ldots, j\}} \sigma_{j}^{2}+\sigma_{X}^{2} \sigma_{j}^{2}\right)\left(\sigma_{X}^{2} d_{\{j+1\}}-d_{\{j+1\}} \sigma_{j}^{2}+\sigma_{X}^{2} \sigma_{j}^{2}\right)}\right), \quad j=1, \ldots, L-1 .
$$

1) If $\max \left\{d_{\{1, \ldots, j\}}, d_{\{j+1\}}\right\}<\sigma_{X}^{2}$, then $d_{\{1, \ldots, j+1\}}^{\diamond}>$ $d_{\{1, \ldots, j+1\}}^{\star}$ and the maximizers to (18) are given by

$$
\sigma_{j}^{2}= \begin{cases}0, & d_{\{1, \ldots, j+1\}} \geq d_{\{1, \ldots, j+1\}}^{\diamond} \\ \sigma_{X}^{2}, & d_{\{1, \ldots, j+1\}} \leq d_{\{1, \ldots, j+1\}}^{\star} \\ \hat{\sigma}_{j}^{2}, & \text { otherwise } \\ & j=1, \ldots, L-1\end{cases}
$$

where $\hat{\sigma}_{j}^{2}$, given at the bottom of the page, is the unique solution to the following equation:

$$
\begin{aligned}
& \left(\frac{\sigma_{X}^{2} d_{\{1, \ldots, j+1\}}}{\sigma_{X}^{2}-d_{\{1, \ldots, j+1\}}}+\hat{\sigma}_{j}^{2}\right)^{-1} \\
& =\left(\frac{\sigma_{X}^{2} d_{\{1, \ldots, j\}}}{\sigma_{X}^{2}-d_{\{1, \ldots, j\}}}+\hat{\sigma}_{j}^{2}\right)^{-1} \\
& \quad+\left(\frac{\sigma_{X}^{2} d_{\{j+1\}}}{\sigma_{X}^{2}-d_{\{j+1\}}}+\hat{\sigma}_{j}^{2}\right)^{-1}
\end{aligned}
$$

for $\hat{\sigma}_{j}^{2} \in\left(0, \sigma_{X}^{2}\right)$. The maximizers $\sigma_{j}^{2}, j=1, \ldots, L-$ 1 , given in (19) are monotonically increasing continuous functions of $d_{\{1, \ldots, j\}}$ and monotonically decreasing continuous functions of $d_{\{1, \ldots, j+1\}}$; furthermore, the monotonicity is strict when $\sigma_{j}^{2} \in\left(0, \sigma_{X}^{2}\right)$.

2) If $\max \left\{d_{\{1, \ldots, j\}}, d_{\{j+1\}}\right\}=\sigma_{X}^{2}$, then $d_{\{1, \ldots, j+1\}}^{\diamond}=$ $d_{\{1, \ldots, j+1\}}^{\star}=\min \left\{d_{\{1, \ldots, j\}}, d_{\{j+1\}}\right\}$ and the maximizers to (18) are given by (20) shown at the bottom of the page. Proof: See Appendix B.

Lemma 3: There exist $\tilde{d}_{\{i\}}, i=1, \ldots, L$, and $\tilde{d}_{\{1, \ldots, i\}}, i=$ $2, \ldots, L$, with

$$
\begin{aligned}
& 0<\tilde{d}_{\{i\}} \leq d_{\{i\}}, \quad i=1, \ldots, L \\
& 0<\tilde{d}_{\{1, \ldots, i\}} \leq d_{\{1, \ldots, i\}}, \quad i=2, \ldots, L \\
& \tilde{d}_{\{1, \ldots, i-1\}}+\tilde{d}_{\{i\}}-\sigma_{X}^{2} \leq \tilde{d}_{\{1, \ldots, i\}} \\
& \quad \leq\left(\frac{1}{\tilde{d}_{\{1, \ldots, i-1\}}}+\frac{1}{\tilde{d}_{\{i\}}}-\frac{1}{\sigma_{X}^{2}}\right)^{-1}, \quad i=2, \ldots, L
\end{aligned}
$$

such that

$$
\max _{\sigma_{j}^{2} \in\left[0, \sigma_{X}^{2}\right], j=1, \ldots, L-1} \psi\left(\tilde{d}_{\{1\}}, \ldots, \tilde{d}_{\{L\}}, \tilde{d}_{\{1,2\}}, \ldots,\right.
$$

$$
\begin{aligned}
& \left.\tilde{d}_{\{1, \ldots, L\}}, \sigma_{1}^{2}, \ldots, \sigma_{L-1}^{2}\right) \\
& \leq \max _{\sigma_{j}^{2} \in\left[0, \sigma_{X}^{2}\right], j=1, \ldots, L-1} \psi\left(d_{\{1\}}, \ldots, d_{\{L\}},\right. \\
& \left.\quad d_{\{1,2\}}, \ldots, d_{\{1, \ldots, L\}}, \sigma_{1}^{2}, \ldots, \sigma_{L-1}^{2}\right) .
\end{aligned}
$$

Proof: See Appendix C.

In view of Lemma 3, to prove (17), it suffices to show that

$$
\begin{aligned}
& \kappa\left(\tilde{d}_{\{1\}}, \ldots, \tilde{d}_{\{L\}}, \tilde{d}_{\{1,2\}}, \ldots, \tilde{d}_{\{1, \ldots, L\}}\right) \\
& \quad \leq \psi\left(\tilde{d}_{\{1\}}, \ldots, \tilde{d}_{\{L\}}, \tilde{d}_{\{1,2\}}, \ldots, \tilde{d}_{\{1, \ldots, L\}}, \tilde{\sigma}_{1}^{2}, \ldots, \tilde{\sigma}_{L-1}^{2}\right)
\end{aligned}
$$

where $\tilde{\sigma}_{j}^{2}, j=1, \ldots, L-1$, are the maximizers to (22).

Now we shall associate $\psi\left(\tilde{d}_{\{1\}}, \ldots, \tilde{d}_{\{L\}}\right.$, $\left.\tilde{d}_{\{1,2\}}, \ldots, \tilde{d}_{\{1, \ldots, L\}}, \quad \tilde{\sigma}_{1}^{2}, \ldots, \tilde{\sigma}_{L-1}^{2}\right)$ with a natural extension of the EGC achievable rate region. For any random variables $U_{1}, \ldots, U_{L}$ jointly distributed with the generic source variable $X$, define

$$
\begin{aligned}
& \mathcal{R}\left(U_{1}, \ldots, U_{L}\right)=\left\{\left(R_{1}, \ldots, R_{L}\right): \sum_{i \in \mathcal{A}} R_{i} \geq\right. \\
&\left.\sum_{i \in \mathcal{A}} h\left(U_{i}\right)-h\left(U_{j}, j \in \mathcal{A} \mid X\right), \emptyset \subset \mathcal{A} \subseteq\{1, \ldots, L\}\right\} .
\end{aligned}
$$

Note that $\mathcal{R}\left(U_{1}, \ldots, U_{L}\right)$ can be viewed as a generalization of the well-known EGC rate region for the 2-description case.

It is observed in [7], [9] that $\mathcal{R}\left(U_{1}, \ldots, U_{L}\right)$ has an interesting contra-polymatroid structure; as a consequence, its vertices and supporting hyperplanes can be easily characterized [11]. Specifically, $\left(R_{1}(\pi), \ldots, R_{L}(\pi)\right)$ is a vertex of $\mathcal{R}\left(U_{1}, \ldots, U_{L}\right)$ for every permutation $\pi$ on $\{1, \ldots, L\}$, where

$$
\begin{aligned}
& R_{\pi(1)}(\pi)=I\left(X ; U_{\pi(1)}\right), \\
& R_{\pi(i)}(\pi)=I\left(X, U_{\pi(1)}, \ldots, U_{\pi(i-1)} ; U_{\pi(i)}\right), \quad i=2, \ldots, L .
\end{aligned}
$$

Moreover, for any nonnegative numbers $\lambda_{1}, \ldots, \lambda_{L}$

$$
\min _{\left(R_{1}, \ldots, R_{L}\right) \in \mathcal{R}\left(U_{1}, \ldots, U_{L}\right)} \sum_{i=1}^{L} \lambda_{i} R_{i}=\sum_{i=1}^{L} \lambda_{\pi(i)} R_{\pi(i)}(\pi)
$$

where $\pi$ is any permutation on $\{1, \ldots, L\}$ such that $\lambda_{\pi(1)} \geq$ $\cdots \geq \lambda_{\pi(L)}$

$$
\hat{\sigma}_{j}^{2}=\sqrt{\left(\frac{\sigma_{X}^{2} d_{\{1, \ldots, j\}}}{\sigma_{X}^{2}-d_{\{1, \ldots, j\}}}-\frac{\sigma_{X}^{2} d_{\{1, \ldots, j+1\}}}{\sigma_{X}^{2}-d_{\{1, \ldots, j+1\}}}\right)\left(\frac{\sigma_{X}^{2} d_{\{j+1\}}}{\sigma_{X}^{2}-d_{\{j+1\}}}-\frac{\sigma_{X}^{2} d_{\{1, \ldots, j+1\}}}{\sigma_{X}^{2}-d_{\{1, \ldots, j+1\}}}\right)}-\frac{\sigma_{X}^{2} d_{\{1, \ldots, j+1\}}}{\sigma_{X}^{2}-d_{\{1, \ldots, j+1\}}}
$$

$$
\sigma_{j}^{2}=\left\{\begin{array}{ll}
0, & d_{\{1, \ldots, j+1\}}>\min \left\{d_{\{1, \ldots, j\}}, d_{\{j+1\}}\right\} \\
\sigma_{X}^{2}, & d_{\{1, \ldots, j+1\}}<\min \left\{d_{\{1, \ldots, j\}}, d_{\{j+1\}}\right\} \\
\text { any number in }\left[0, \sigma_{X}^{2}\right], & \text { otherwise }
\end{array} \quad j=1, \ldots, L-1 .\right.
$$


Since it is assumed that $\alpha_{1} \geq \cdots \geq \alpha_{L}>0$, we have

$$
\begin{aligned}
& \min _{\left(R_{1}, \ldots, R_{L}\right) \in \mathcal{R}\left(U_{1}, \ldots, U_{L}\right)} \sum_{i=1}^{L} \alpha_{i} R_{i} \\
= & \alpha_{1} I\left(X ; U_{1}\right)+\sum_{i=2}^{L} \alpha_{i} I\left(X, U_{1}, \ldots, U_{i-1} ; U_{i}\right) \\
= & \left(\alpha_{1}-\alpha_{2}\right) I\left(X ; U_{1}\right) \\
& +\sum_{i=2}^{L}\left(\alpha_{i}-\alpha_{i+1}\right)\left(I\left(X ; U_{1}\right)\right. \\
& \left.+\sum_{j=1}^{i-1} I\left(X, U_{1}, \ldots, U_{j} ; U_{j+1}\right)\right)
\end{aligned}
$$

where $\alpha_{L+1} \triangleq 0$. Note that

$$
\begin{aligned}
I\left(X ; U_{1}\right)+\sum_{j=1}^{i-1} I\left(X, U_{1}, \ldots, U_{j} ; U_{j+1}\right) \\
=I\left(X ; U_{1}\right)+\sum_{j=1}^{i-1}\left(I\left(U_{1}, \ldots, U_{j} ; U_{j+1}\right)\right. \\
\left.\quad+I\left(X ; U_{j+1} \mid U_{1}, \ldots, U_{j}\right)\right) \\
=I\left(X ; U_{1}, \ldots, U_{i}\right)+\sum_{j=1}^{i-1} I\left(U_{1}, \ldots, U_{j} ; U_{j+1}\right) .
\end{aligned}
$$

Substituting (24) into (23), we obtain

$$
\begin{aligned}
& \min _{\left(R_{1}, \ldots, R_{L}\right) \in \mathcal{R}\left(U_{1}, \ldots, U_{L}\right)} \sum_{i=1}^{L} \alpha_{i} R_{i} \\
= & \left(\alpha_{1}-\alpha_{2}\right) I\left(X ; U_{1}\right)+\sum_{i=2}^{L}\left(\alpha_{i}-\alpha_{i+1}\right) \\
& \times\left(I\left(X ; U_{1}, \ldots, U_{i}\right)+\sum_{j=1}^{i-1} I\left(U_{1}, \ldots, U_{j} ; U_{j+1}\right)\right) \\
= & \sum_{i=1}^{L-1}\left(\left(\alpha_{i}-\alpha_{i+1}\right) I\left(X ; U_{1}, \ldots, U_{i}\right)\right. \\
& \left.+\alpha_{i+1} I\left(U_{1}, \ldots, U_{i} ; U_{i+1}\right)\right)+\alpha_{L} I\left(X ; U_{1}, \ldots, U_{L}\right) .
\end{aligned}
$$

It can be readily shown (cf. [2]) that

$$
\begin{aligned}
\kappa\left(\tilde{d}_{\{1\}}, \ldots, \tilde{d}_{\{L\}}, \tilde{d}_{\{1,2\}}, \ldots, \tilde{d}_{\{1, \ldots, L\}}\right) & \\
& \geq \min _{\left(R_{1}, \ldots, R_{L}\right) \in \mathcal{R}\left(U_{1}, \ldots, U_{L}\right)} \sum_{i=1}^{L} \alpha_{i} R_{i}
\end{aligned}
$$

for $\left(U_{1}, \ldots, U_{L}\right)$ with

$$
\begin{aligned}
& \operatorname{cov}\left(X \mid U_{i}\right) \leq \tilde{d}_{\{i\}}, \quad i=1, \ldots, L \\
& \operatorname{cov}\left(X \mid U_{1}, \ldots, U_{i}\right) \leq \tilde{d}_{\{1, \ldots, i\}}, \quad i=2, \ldots, L .
\end{aligned}
$$

Therefore, to complete the proof, it suffices to construct $\left(U_{1}, \ldots, U_{L}\right)$ satisfying $(26)$ and (27) such that

$$
\begin{aligned}
& \min _{\left(R_{1}, \ldots, R_{L}\right) \in \mathcal{R}\left(U_{1}, \ldots, U_{L}\right)} \sum_{i=1}^{L} \alpha_{i} R_{i}=\psi\left(\tilde{d}_{\{1\}}, \ldots,\right. \\
&\left.\tilde{d}_{\{L\}}, \tilde{d}_{\{1,2\}}, \ldots, \tilde{d}_{\{1, \ldots, L\}}, \tilde{\sigma}_{1}^{2}, \ldots, \tilde{\sigma}_{L-1}^{2}\right) .
\end{aligned}
$$

It is easy to verify the following lemma, which will be served as a building block for constructing the desired covariance matrix of $\left(U_{1}, \ldots, U_{L}\right)$.

Lemma 4: For any $d_{0}, d_{1}, d_{2} \in\left(0, \sigma_{X}^{2}\right)$ satisfying

$$
d_{1}+d_{2}-\sigma_{X}^{2} \leq d_{0} \leq\left(\frac{1}{d_{1}}+\frac{1}{d_{2}}-\frac{1}{\sigma_{X}^{2}}\right)^{-1}
$$

define

$$
\begin{aligned}
& V_{1}=X+N+\sqrt{\frac{\sigma_{X}^{2} d_{1}}{\sigma_{X}^{2}-d_{1}}-\frac{\sigma_{X}^{2} d_{0}}{\sigma_{X}^{2}-d_{0}}} N^{\prime}, \\
& V_{2}=X+N-\sqrt{\frac{\sigma_{X}^{2} d_{2}}{\sigma_{X}^{2}-d_{2}}-\frac{\sigma_{X}^{2} d_{0}}{\sigma_{X}^{2}-d_{0}}} N^{\prime}
\end{aligned}
$$

where $N \sim \mathcal{N}\left(0, \frac{\sigma_{X}^{2} d_{0}}{\sigma_{X}^{2}-d_{0}}\right), N^{\prime} \sim \mathcal{N}(0,1)$, and $X \sim \mathcal{N}\left(0, \sigma_{X}^{2}\right)$ are independent. We have

$$
\begin{aligned}
& \operatorname{cov}\left(X \mid V_{i}\right)=d_{i}, \quad i=1,2, \\
& \operatorname{cov}\left(X \mid V_{1}, V_{2}\right)=\operatorname{cov}(X \mid X+N)=d_{0}, \\
& \mathbb{E}\left(V_{1} V_{2}\right) \in\left[0, \sigma_{X}^{2}\right] .
\end{aligned}
$$

Remark:

1) Given $V_{1}$ and $V_{2}$, one can construct $X+N$.

2) Write $X=\hat{X}+\tilde{X}$, where $\hat{X} \sim \mathcal{N}\left(0, \sigma_{X}^{2}-\sigma^{2}\right)$ and $\tilde{X} \sim \mathcal{N}\left(0, \sigma^{2}\right)$ are independent, and

$$
\begin{array}{r}
\sigma^{2}=\sqrt{\left(\frac{\sigma_{X}^{2} d_{1}}{\sigma_{X}^{2}-d_{1}}-\frac{\sigma_{X}^{2} d_{0}}{\sigma_{X}^{2}-d_{0}}\right)\left(\frac{\sigma_{X}^{2} d_{2}}{\sigma_{X}^{2}-d_{2}}-\frac{\sigma_{X}^{2} d_{0}}{\sigma_{X}^{2}-d_{0}}\right)} \\
-\frac{\sigma_{X}^{2} d_{0}}{\sigma_{X}^{2}-d_{0}} .
\end{array}
$$

Note that $V_{1}$ and $V_{2}$ are independent conditioned on $\hat{X}$ since

$$
\begin{aligned}
& \mathbb{E}\left(\left(\tilde{X}+N+\sqrt{\frac{\sigma_{X}^{2} d_{1}}{\sigma_{X}^{2}-d_{1}}-\frac{\sigma_{X}^{2} d_{0}}{\sigma_{X}^{2}-d_{0}}} N^{\prime}\right)\right. \\
& \left.\left(\tilde{X}+N-\sqrt{\frac{\sigma_{X}^{2} d_{1}}{\sigma_{X}^{2}-d_{1}}-\frac{\sigma_{X}^{2} d_{0}}{\sigma_{X}^{2}-d_{0}}} N^{\prime}\right)\right)=0 .
\end{aligned}
$$

Therefore, we have

$$
\begin{aligned}
I\left(V_{1} ; V_{2}\right) \\
=I\left(\hat{X} ; V_{1}\right)+I\left(\hat{X} ; V_{2}\right)+I\left(V_{1} ; V_{2} \mid \hat{X}\right) \\
\quad-I\left(\hat{X} ; V_{1}, V_{2}\right) \\
=I\left(\hat{X} ; V_{1}\right)+I\left(\hat{X} ; V_{2}\right)-I\left(\hat{X} ; V_{1}, V_{2}\right)
\end{aligned}
$$




$$
\begin{aligned}
= & I\left(\hat{X} ; V_{1}\right)+I\left(\hat{X} ; V_{2}\right)-I(\hat{X} ; X+N) \\
= & \frac{1}{2} \log \\
& \left(\frac{\sigma_{X}^{4}\left(\sigma_{X}^{2} d_{0}-d_{0} \sigma^{2}+\sigma_{X}^{2} \sigma^{2}\right)}{\left(\sigma_{X}^{2} d_{1}-d_{1} \sigma^{2}+\sigma_{X}^{2} \sigma^{2}\right)\left(\sigma_{X}^{2} d_{2}-d_{2} \sigma^{2}+\sigma_{X}^{2} \sigma^{2}\right)}\right) .
\end{aligned}
$$

Now we proceed to give an explicit construction of $\left(U_{1}, \ldots, U_{L}\right)$. First consider the case where $\tilde{d}_{\{i\}}<\sigma_{X}^{2}$ for all $i=1, \ldots, L$, which, by (21), further implies $d_{\{1, \ldots, i\}}<\sigma_{X}^{2}$ for all $i=2, \ldots, L$. In view of (21) and Lemma 2 , we have the first equation shown at the bottom of the page.

By Lemma 4, we can construct

$$
\begin{aligned}
& U_{1}=X+N_{\{1,2\}}+\sqrt{\frac{\sigma_{X}^{2} \tilde{d}_{\{1\}}}{\sigma_{X}^{2}-\tilde{d}_{\{1\}}}-\frac{\sigma_{X}^{2} \tilde{d}_{\{1,2\}}}{\sigma_{X}^{2}-\tilde{d}_{\{1,2\}}}} N_{\{1,2\}}^{\prime}, \\
& U_{2}=X+N_{\{1,2\}}-\sqrt{\frac{\sigma_{X}^{2} \tilde{d}_{\{2\}}}{\sigma_{X}^{2}-\tilde{d}_{\{2\}}}-\frac{\sigma_{X}^{2} \tilde{d}_{\{1,2\}}}{\sigma_{X}^{2}-\tilde{d}_{\{1,2\}}}} N_{\{1,2\}}^{\prime}
\end{aligned}
$$

where $N_{\{1,2\}} \sim \mathcal{N}\left(0, \frac{\sigma_{X}^{2} \tilde{d}_{\{1,2\}}}{\sigma_{X}^{2}-\tilde{d}_{\{1,2\}}}\right), N_{\{1,2\}}^{\prime} \sim \mathcal{N}(0,1)$, and $X \sim$ $\mathcal{N}\left(0, \sigma_{X}^{2}\right)$ are mutually independent. Now we write

$N_{\{1,2\}}=N_{\{1,2,3\}}+\sqrt{\frac{\sigma_{X}^{2} \tilde{d}_{\{1,2\}}}{\sigma_{X}^{2}-\tilde{d}_{\{1,2\}}}-\frac{\sigma_{X}^{2} \tilde{d}_{\{1,2,3\}}}{\sigma_{X}^{2}-\tilde{d}_{\{1,2,3\}}}} N_{\{1,2,3\}}^{\prime}$

where $N_{\{1,2,3\}} \sim \mathcal{N}\left(0, \frac{\sigma_{X}^{2} \tilde{d}_{\{1,2,3\}}}{\sigma_{X}^{2}-\tilde{d}_{\{1,2,3\}}}\right)$ and $N_{\{1,2,3\}}^{\prime} \sim \mathcal{N}(0,1)$ are mutually independent. Again, by Lemma 4, we can construct $U_{3}=X+N_{\{1,2,3\}}-\sqrt{\frac{\sigma_{X}^{2} \tilde{d}_{\{3\}}}{\sigma_{X}^{2}-\tilde{d}_{\{3\}}}-\frac{\sigma_{X}^{2} \tilde{d}_{\{1,2,3\}}}{\sigma_{X}^{2}-\tilde{d}_{\{1,2,3\}}}} N_{\{1,2,3\}}^{\prime}$.

Now we can further write

$N_{\{1,2,3\}}=N_{\{1,2,3,4\}}$

$$
+\sqrt{\frac{\sigma_{X}^{2} \tilde{d}_{\{1,2,3\}}}{\sigma_{X}^{2}-\tilde{d}_{\{1,2,3\}}}-\frac{\sigma_{X}^{2} \tilde{d}_{\{1,2,3,4\}}}{\sigma_{X}^{2}-\tilde{d}_{\{1,2,3,4\}}}} N_{\{1,2,3,4\}}^{\prime}
$$

where $N_{\{1,2,3,4\}} \sim \mathcal{N}\left(0, \frac{\sigma_{X}^{2} \tilde{d}_{\{1,2,3,4\}}}{\sigma_{X}^{2}-\tilde{d}_{\{1,2,3,4\}}}\right)$ and $N_{\{1,2,3,4\}}^{\prime} \sim$ $\mathcal{N}(0,1)$ are mutually independent. By successively applying this procedure, we obtain

$$
\begin{aligned}
& U_{1}= X+N_{\{1,2\}} \\
&+\sqrt{\frac{\sigma_{X}^{2} \tilde{d}_{\{1\}}}{\sigma_{X}^{2}-\tilde{d}_{\{1\}}}-\frac{\sigma_{X}^{2} \tilde{d}_{\{1,2\}}}{\sigma_{X}^{2}-\tilde{d}_{\{1,2\}}}} N_{\{1,2\}}^{\prime}, \\
& U_{i}= X+N_{\{1, \ldots, i\}} \\
&-\sqrt{\frac{\sigma_{X}^{2} \tilde{d}_{\{i\}}}{\sigma_{X}^{2}-\tilde{d}_{\{i\}}}-\frac{\sigma_{X}^{2} \tilde{d}_{\{1, \ldots, i\}}}{\sigma_{X}^{2}-\tilde{d}_{\{1, \ldots, i\}}}} N_{\{1, \ldots, i\}}^{\prime}, \\
& i=2, \ldots, L
\end{aligned}
$$

with the property (29)-(32) shown at the bottom of the page. It is easy to see that the distortion constraints (26) and (27) are satisfied by the constructed $\left(U_{1}, \ldots, U_{L}\right)$. Moreover, one can readily verify (28) by substituting (29)-(32) into (25).

Now consider the general scenario in which $\tilde{d}_{\{i\}}<\sigma_{X}^{2}, i \in$ $\mathcal{A}$, and $\tilde{d}_{\{i\}}=\sigma_{X}^{2}, i \notin \mathcal{A}$, for some $\mathcal{A} \subseteq\{1, \ldots, L\}$. In this case, one can set $U_{i}=0$ for $i \notin \mathcal{A}$ and apply the previous construction to $U_{i}, i \in \mathcal{A}$. The proof is complete.

\section{SAdDle Point Analysis: Proof of Theorem 3}

In order to show that max-min [cf. (3)] and min-max (cf. (4)) coincide, it suffices to prove the existence of a saddle point.

Given $\sigma_{j}^{2}, j=1, \ldots, L-1$, the minimization problem

$$
\begin{aligned}
& d_{\{1, \ldots, i\}} \in\left[0, d_{\{1, \ldots, i\}}^{\prime}\right], i=2, \ldots, L-1 \\
& \psi\left(d_{\{1\}}, \ldots, d_{\{L\}}, d_{\{1,2\}}, \ldots, d_{\{1, \ldots, L\}}, \sigma_{1}^{2}, \ldots, \sigma_{L-1}^{2}\right)
\end{aligned}
$$

$$
\begin{aligned}
& \tilde{\sigma}_{j}^{2}=\sqrt{\left(\frac{\sigma_{X}^{2} \tilde{d}_{\{1, \ldots, j\}}}{\sigma_{X}^{2}-\tilde{d}_{\{1, \ldots, j\}}}-\frac{\sigma_{X}^{2} \tilde{d}_{\{1, \ldots, j+1\}}}{\sigma_{X}^{2}-\tilde{d}_{\{1, \ldots, j+1\}}}\right)\left(\frac{\sigma_{X}^{2} \tilde{d}_{\{j+1\}}}{\sigma_{X}^{2}-\tilde{d}_{\{j+1\}}}-\frac{\sigma_{X}^{2} \tilde{d}_{\{1, \ldots, j+1\}}}{\sigma_{X}^{2}-\tilde{d}_{\{1, \ldots, j+1\}}}\right)}-\frac{\sigma_{X}^{2} \tilde{d}_{\{1, \ldots, j+1\}}}{\sigma_{X}^{2}-\tilde{d}_{\{1, \ldots, j+1\}}}, \\
& j=1, \ldots, L-1 \text {. }
\end{aligned}
$$

$$
\begin{aligned}
& I\left(X ; U_{1}\right)=\frac{1}{2} \log \left(\frac{\sigma_{X}^{2}}{\tilde{d}_{\{1\}}}\right) \\
& I\left(X ; U_{1}, \ldots, U_{i}\right)=I\left(X ; X+N_{\{1, \ldots, i\}}\right)=\frac{1}{2} \log \left(\frac{\sigma_{X}^{2}}{\tilde{d}_{\{1, \ldots, i\}}}\right), \quad i=2, \ldots, L \\
& I\left(U_{1} ; U_{2}\right)=\frac{1}{2} \log \left(\frac{\sigma_{X}^{4}\left(\sigma_{X}^{2} \tilde{d}_{\{1,2\}}-\tilde{d}_{\{1,2\}} \tilde{\sigma}_{1}^{2}+\sigma_{X}^{2} \tilde{\sigma}_{1}^{2}\right)}{\left(\sigma_{X}^{2} \tilde{d}_{\{1\}}-\tilde{d}_{\{1\}} \tilde{\sigma}_{1}^{2}+\sigma_{X}^{2} \tilde{\sigma}_{1}^{2}\right)\left(\sigma_{X}^{2} \tilde{d}_{\{2\}}-\tilde{d}_{\{2\}} \tilde{\sigma}_{1}^{2}+\sigma 2_{X} \tilde{\sigma}_{1}^{2}\right)}\right) \\
& I\left(U_{1}, \ldots, U_{i-1} ; U_{i}\right)=I\left(X+N_{\{1, \ldots, i-1\}} ; U_{i}\right) \\
& \quad=\frac{1}{2} \log \left(\frac{\sigma_{X}^{4}\left(\sigma_{X}^{2} \tilde{d}_{\{1, \ldots, i\}}-\tilde{d}_{\{1, \ldots, i\}} \tilde{\sigma}_{i-1}^{2}+\sigma_{X}^{2} \tilde{\sigma}_{i-1}^{2}\right)}{\left(\sigma_{X}^{2} \tilde{d}_{\{1, \ldots, i-1\}}-\tilde{d}_{\{1, \ldots, i-1\}} \tilde{\sigma}_{i-1}^{2}+\sigma_{X}^{2} \tilde{\sigma}_{i-1}^{2}\right)\left(\sigma_{X}^{2} \tilde{d}_{\{i\}}-\tilde{d}_{\{i\}} \tilde{\sigma}_{i-1}^{2}+\sigma_{X}^{2} \tilde{\sigma}_{i-1}^{2}\right)}\right), \quad i=2, \ldots, L .
\end{aligned}
$$


can be decomposed into

$$
\begin{aligned}
& \min _{\{1, \ldots, i\}} \in\left[0, d_{\{1, \ldots, i\}}^{\prime}\right] \\
& \frac{\alpha_{i}}{2} \log \left(\sigma_{X}^{2} d_{\{1, \ldots, i\}}-d_{\{1, \ldots, i\}} \sigma_{i-1}^{2}+\sigma_{X}^{2} \sigma_{i-1}^{2}\right) \\
& -\frac{\alpha_{i}-\alpha_{i+1}}{2} \log \left(d_{\{1, \ldots, i\}}\right) \\
& -\frac{\alpha_{i+1}}{2} \log \left(\sigma_{X}^{2} d_{\{1, \ldots, i\}}-d_{\{1, \ldots, i\}} \sigma_{i}^{2}+\sigma_{X}^{2} \sigma_{i}^{2}\right) \\
& i=2, \ldots, L-1 .
\end{aligned}
$$

Lemma 5: If $\alpha_{i}>\alpha_{i+1}$, then the minimizers to (33) are given by (34) shown at the bottom of the page, where

$$
\hat{d}_{\{1, \ldots, i\}}=\frac{\left(\alpha_{i}-\alpha_{i+1}\right) \sigma_{X}^{2} \sigma_{i-1}^{2} \sigma_{i}^{2}}{\alpha_{i+1} \sigma_{i}^{2}\left(\sigma_{X}^{2}-\sigma_{i-1}^{2}\right)-\alpha_{i} \sigma_{i-1}^{2}\left(\sigma_{X}^{2}-\sigma_{i}^{2}\right)} .
$$

If $\alpha_{i}=\alpha_{i+1}$, then the minimizers to (33) are given by

$$
d_{\{1, \ldots, i\}}=\left\{\begin{array}{lr}
d_{\{1, \ldots, i\}}^{\prime}, & \sigma_{i-1}^{2}>\sigma_{i}^{2} \\
0 & \sigma_{i-1}^{2}<\sigma_{i}^{2} \\
\text { any number in }\left[0, d_{\{1, \ldots, i\}}^{\prime}\right], & \sigma_{i-1}^{2}=\sigma_{i}^{2} \\
i=2, \ldots, L-1 .
\end{array}\right.
$$

\section{Proof: See Appendix D.}

First consider the case where individual distortion constraints $d_{\{i\}}, i=1, \ldots, L$, auxiliary distortion constraints $d_{\{1, \ldots, i\}}^{\prime}, i=$ $2, \ldots, L-1$, and central distortion constraint $d_{\{1, \ldots, L\}}$ are all less than $\sigma_{X}^{2}$.

Let $\left(\tilde{d}_{\{1,2\}}, \ldots, \tilde{d}_{\{1, \ldots, L-1\}}, \tilde{\sigma}_{1}^{2}, \ldots, \tilde{\sigma}_{L-1}^{2}\right)$ be a solution to the min-max game in (4). We shall show that $\left(\tilde{d}_{\{1,2\}}, \ldots, \tilde{d}_{\{1, \ldots, L-1\}}, \tilde{\sigma}_{1}^{2}, \ldots, \tilde{\sigma}_{L-1}^{2}\right)$ must satisfy the saddle point conditions (19) and (34) [or (35)].

Note that (19) is automatically satisfied. Suppose (34) [or (35)] is violated by some $\tilde{d}_{\left\{1, \ldots, i^{*}\right\}}$. We shall focus on $\tilde{d}_{\left\{1, \ldots, i^{*}\right\}}$, $\tilde{\sigma}_{i^{*}-1}^{2}$, and $\tilde{\sigma}_{i^{*}}^{2}$ while assuming everything else is fixed. Since $\tilde{\sigma}_{i^{*}-1}^{2}$ and $\tilde{\sigma}_{i^{*}}^{2}$ are determined by $\tilde{d}_{\left\{1, \ldots, i^{*}\right\}}$ according to (19), to stress this dependence, we denote $\tilde{\sigma}_{i^{*}-1}^{2}$ and $\tilde{\sigma}_{i^{*}}^{2}$ as $\sigma_{i^{*}-1}^{2}\left(\tilde{d}_{\left\{1, \ldots, i^{*}\right\}}\right)$ and $\sigma_{i^{*}}^{2}\left(\tilde{d}_{\left\{1, \ldots, i^{*}\right\}}\right)$, respectively. By Lemma $2, \sigma_{i^{*}-1}^{2}\left(\tilde{d}_{\left\{1, \ldots, i^{*}\right\}}\right)$ is a monotonically decreasing continuous function of $\tilde{d}_{\left\{1, \ldots, i^{*}\right\}}$ while $\sigma_{i^{*}}^{2}\left(\tilde{d}_{\left\{1, \ldots, i^{*}\right\}}\right)$ is a monotonically increasing continuous function of $\tilde{d}_{\left\{1, \ldots, i^{*}\right\}}$. Define

$$
\begin{aligned}
& \eta\left(\tilde{d}_{\left\{1, \ldots, i^{*}\right\}}\right) \\
& =\alpha_{i^{*}+1} \sigma_{i^{*}}^{2}\left(\tilde{d}_{\left\{1, \ldots, i^{*}\right\}}\right)\left(\sigma_{X}^{2}-\sigma_{i^{*}-1}^{2}\left(\tilde{d}_{\left\{1, \ldots, i^{*}\right\}}\right)\right) \\
& \quad-\alpha_{i^{*}} \sigma_{i^{*}-1}^{2}\left(\tilde{d}_{\left\{1, \ldots, i^{*}\right\}}\right)\left(\sigma_{X}^{2}-\sigma_{i^{*}}^{2}\left(\tilde{d}_{\left\{1, \ldots, i^{*}\right\}}\right)\right) .
\end{aligned}
$$

Note that $\eta\left(\tilde{d}_{\left\{1, \ldots, i^{*}\right\}}\right)$ is a monotonically increasing continuous function of $\tilde{d}_{\left\{1, \ldots, i^{*}\right\}}$. Now consider the following cases.

1) Case 1: $\alpha_{i^{*}}>\alpha_{i^{*}+1}, \tilde{d}_{\left\{1, \ldots, i^{*}\right\}}<d_{\left\{1, \ldots, i^{*}\right\}}^{\prime}$, and $\eta\left(\tilde{d}_{\left\{1, \ldots, i^{*}\right\}}\right) \leq 0$. Increase $d_{\left\{1, \ldots, i^{*}\right\}}$ from $\tilde{d}_{\left\{1, \ldots, i^{*}\right\}}$ until one of the following happens: $d_{\left\{1, \ldots, i^{*}\right\}}=d_{\left\{1, \ldots, i^{*}\right\}}^{\prime}$ or $\eta\left(d_{\left\{1, \ldots, i^{*}\right\}}\right)=0$. If we first have $d_{\left\{1, \ldots, i^{*}\right\}}=d_{\left\{1, \ldots, i^{*}\right\}}^{\prime}$, then set $d_{\left\{1, \ldots, i^{*}\right\}}^{*}=d_{\left\{1, \ldots, i^{*}\right\}}^{\prime}$. Now suppose we first have $\eta\left(d_{\left\{1, \ldots, i^{*}\right\}}\right)=0$. Denote the corresponding $d_{\left\{1, \ldots, i^{*}\right\}}$ by $\bar{d}_{\left\{1, \ldots, i^{*}\right\}}$. It can be seen that only the following three situations are possible:

a) $\sigma_{i^{*}-1}^{2}\left(\bar{d}_{\left\{1, \ldots, i^{*}\right\}}\right)=\sigma_{i^{*}}^{2}\left(\bar{d}_{\left\{1, \ldots, i^{*}\right\}}\right)=0$,

b) $\sigma_{i^{*}-1}^{2}\left(\bar{d}_{\left\{1, \ldots, i^{*}\right\}}\right)=\sigma_{i^{*}}^{2}\left(\bar{d}_{\left\{1, \ldots, i^{*}\right\}}\right)=\sigma_{X}^{2}$,

c) $\sigma_{i^{*}-1}^{2}\left(\bar{d}_{\left\{1, \ldots, i^{*}\right\}}\right) \in\left(0, \sigma_{X}^{2}\right)$ and $\sigma_{i^{*}}^{2}\left(\bar{d}_{\left\{1, \ldots, i^{*}\right\}}\right) \in$ $\left(0, \sigma_{X}^{2}\right)$

For situation a), we set $d_{\left\{1, \ldots, i^{*}\right\}}^{*}=\bar{d}_{\left\{1, \ldots, i^{*}\right\}}$. For situation $b$ ), we further increase $d_{\left\{1, \ldots, i^{*}\right\}}$ until one of the following happens: $d_{\left\{1, \ldots, i^{*}\right\}}=d_{\left\{1, \ldots, i^{*}\right\}}^{\prime}$ or $\eta\left(d_{\left\{1, \ldots, i^{*}\right\}}\right)$ becomes positive. If $d_{\left\{1, \ldots, i^{*}\right\}}=d_{\left\{1, \ldots, i^{*}\right\}}^{\prime}$ happens first, then set $d_{\left\{1, \ldots, i^{*}\right\}}^{*}=d_{\left\{1, \ldots, i^{*}\right\}}^{\prime}$. If $\eta\left(d_{\left\{1, \ldots, i^{*}\right\}}\right)$ first becomes positive, then we further increase $d_{\left\{1, \ldots, i^{*}\right\}}$ until it meets $\min \left\{\hat{d}_{\left\{1, \ldots, i^{*}\right\}}, d_{\left\{1, \ldots, i^{*}\right\}}^{\prime}\right\}$ and then set $d_{\left\{1, \ldots, i^{*}\right\}}^{*}$ to be equal to the corresponding $d_{\left\{1, \ldots, i^{*}\right\}}$. For situation c), $\eta\left(d_{\left\{1, \ldots, i^{*}\right\}}\right)$ is at the point of turning positive (cf. Lemma 2). We can further increase $d_{\left\{1, \ldots, i^{*}\right\}}$ until it meets $\min \left\{\hat{d}_{\left\{1, \ldots, i^{*}\right\}}, d_{\left\{1, \ldots, i^{*}\right\}}^{\prime}\right\}$ and then set $d_{\left\{1, \ldots, i^{*}\right\}}^{*}$ to be equal to the corresponding $d_{\left\{1, \ldots, i^{*}\right\}}$. Note that when $\eta\left(d_{\left\{1, \ldots, i^{*}\right\}}\right)>0$, the quantity $\hat{d}_{\left\{1, \ldots, i^{*}\right\}}$ is a monotonically increasing continuous function of $\sigma_{i^{*}-1}^{2}\left(d_{\left\{1, \ldots, i^{*}\right\}}\right)$ and a monotonically decreasing continuous function of $\sigma_{i^{*}}^{2}\left(d_{\left\{1, \ldots, i^{*}\right\}}\right)$, thus, is a monotonically decreasing continuous function of $d_{\left\{1, \ldots, i^{*}\right\}}$. Furthermore, we have $\hat{d}_{\left\{1, \ldots, i^{*}\right\}}=\infty$ at the point where $\eta\left(d_{\left\{1, \ldots, i^{*}\right\}}\right)$ turns positive. Therefore, the above procedure is always possible.

2) Case 2: $\alpha_{i^{*}}>\alpha_{i^{*}+1}, \quad \tilde{d}_{\left\{1, \ldots, i^{*}\right\}}<$ $\min \left\{\hat{d}_{\left\{1, \ldots, i^{*}\right\}}, d_{\left\{1, \ldots, i^{*}\right\}}^{\prime}\right\}$, and $\eta\left(\tilde{d}_{\left\{1, \ldots, i^{*}\right\}}\right)>0$. We can increase $d_{\left\{1, \ldots, i^{*}\right\}}$ from $\tilde{d}_{\left\{1, \ldots, i^{*}\right\}}$ until it hits $\min \left\{\hat{d}_{\left\{1, \ldots, i^{*}\right\}}, d_{\left\{1, \ldots, i^{*}\right\}}^{\prime}\right\}$ and then set $d_{\left\{1, \ldots, i^{*}\right\}}^{*}$ to be equal to the corresponding $d_{\left\{1, \ldots, i^{*}\right\}}$.

3) Case 3: $\alpha_{i^{*}}>\alpha_{i^{*}+1}, \hat{d}_{\left\{1, \ldots, i^{*}\right\}}<\tilde{d}_{\left\{1, \ldots, i^{*}\right\}} \leq d_{\left\{1, \ldots, i^{*}\right\}}^{\prime}$, and $\eta\left(\tilde{d}_{\left\{1, \ldots, i^{*}\right\}}\right)>0$. Decrease $d_{\left\{1, \ldots, i^{*}\right\}}$ from $\tilde{d}_{\left\{1, \ldots, i^{*}\right\}}$ until one of the following happens: $d_{\left\{1, \ldots, i^{*}\right\}}=\hat{d}_{\left\{1, \ldots, i^{*}\right\}}$ or $\eta\left(d_{\left\{1, \ldots, i^{*}\right\}}\right)=0$. If we first have $d_{\left\{1, \ldots, i^{*}\right\}}=\hat{d}_{\left\{1, \ldots, i^{*}\right\}}$, then set $d_{\left\{1, \ldots, i^{*}\right\}}^{*}=\hat{d}_{\left\{1, \ldots, i^{*}\right\}}$. Now suppose that $\eta\left(d_{\left\{1, \ldots, i^{*}\right\}}\right) \stackrel{1}{=} 0$ happens first. Denote the corresponding $d_{\left\{1, \ldots, i^{*}\right\}}$ by $d_{\left\{1, \ldots, i^{*}\right\}}^{*}$. Note that that both

$$
d_{\{1, \ldots, i\}}= \begin{cases}d_{\{1, \ldots, i\}}^{\prime}, & \alpha_{i+1} \sigma_{i}^{2}\left(\sigma_{X}^{2}-\sigma_{i-1}^{2}\right)<\alpha_{i} \sigma_{i-1}^{2}\left(\sigma_{X}^{2}-\sigma_{i}^{2}\right) \\ \min \left\{\hat{d}_{\{1, \ldots, i\}}, d_{\{1, \ldots, i\}}^{\prime}\right\}, & \alpha_{i+1} \sigma_{i}^{2}\left(\sigma_{X}^{2}-\sigma_{i-1}^{2}\right)>\alpha_{i} \sigma_{i-1}^{2}\left(\sigma_{X}^{2}-\sigma_{i}^{2}\right) \\ d_{\{1, \ldots, i\}}^{\prime}, & \alpha_{i+1} \sigma_{i}^{2}\left(\sigma_{X}^{2}-\sigma_{i-1}^{2}\right)=\alpha_{i} \sigma_{i-1}^{2}\left(\sigma_{X}^{2}-\sigma_{i}^{2}\right) \text { and } \sigma_{i-1}^{2} \sigma_{i}^{2}>0 \\ \text { any number in }\left[0, d_{\{1, \ldots, i\}}^{\prime}\right], & \sigma_{i-1}^{2}=\sigma_{i}^{2}=0\end{cases}
$$


$\sigma_{i^{*}-1}^{2}\left(d_{\left\{1, \ldots, i^{*}\right\}}^{*}\right)$ and $\sigma_{i^{*}}^{2}\left(d_{\left\{1, \ldots, i^{*}\right\}}^{*}\right)$ must be equal to zero.

4) Case 4: $\alpha_{i^{*}}=\alpha_{i^{*}+1}, \tilde{d}_{\left\{1, \ldots, i^{*}\right\}}<d_{\left\{1, \ldots, i^{*}\right\}}^{\prime}$, and $\sigma_{i^{*}-1}^{2}\left(\tilde{d}_{\left\{1, \ldots, i^{*}\right\}}\right)>\sigma_{i^{*}}^{2}\left(\tilde{d}_{\left\{1, \ldots, i^{*}\right\}}\right)$. Increase $d_{\left\{1, \ldots, i^{*}\right\}}$ from $d_{\left\{1, \ldots, i^{*}\right\}}$ until one of the following happens: $d_{\left\{1, \ldots, i^{*}\right\}}=d_{\left\{1, \ldots, i^{*}\right\}}^{\prime}$ or $\sigma_{i^{*}-1}^{2}\left(d_{\left\{1, \ldots, i^{*}\right\}}\right)=$ $\sigma_{i^{*}}^{2}\left(d_{\left\{1, \ldots, i^{*}\right\}}\right)$. Set $d_{\left\{1, \ldots, i^{*}\right\}}^{*}$ to be equal to the corresponding $d_{\left\{1, \ldots, i^{*}\right\}}$.

5) Case 5: $\alpha_{i^{*}}=\alpha_{i^{*}+1}, \tilde{d}_{\left\{1, \ldots, i^{*}\right\}}>0$, and $\sigma_{i^{*}-1}^{2}\left(\tilde{d}_{\left\{1, \ldots, i^{*}\right\}}\right)<\sigma_{i^{*}}^{2}\left(\tilde{d}_{\left\{1, \ldots, i^{*}\right\}}\right)$. Decrease $d_{\left\{1, \ldots, i^{*}\right\}}$ from $\tilde{d}_{\left\{1, \ldots, i^{*}\right\}}$ until $\sigma_{i^{*}-1}^{2}\left(d_{\left\{1, \ldots, i^{*}\right\}}\right)=\sigma_{i^{*}}^{2}\left(d_{\left\{1, \ldots, i^{*}\right\}}\right)$ and then set $d_{\left\{1, \ldots, i^{*}\right\}}^{*}$ to be equal to the corresponding $d_{\left\{1, \ldots, i^{*}\right\}}$.

Through the above construction, the resulting $d_{\left\{1, \ldots, b_{i *}\right\}}^{*}$, $\sigma_{i^{*}-1}^{2}\left(d_{\left\{1, \ldots, i^{*}\right\}}^{*}\right)$, and $\sigma_{i^{*}}^{2}\left(d_{\left\{1, \ldots, i^{*}\right\}}^{*}\right)$ must satisfy (34) (or (35)). Note that

$$
\begin{aligned}
& \psi( d_{\{1\}}, \ldots, d_{\{L\}}, \tilde{d}_{\{1,2\}}, \ldots, \\
&\left.\tilde{d}_{\{1, \ldots, L-1\}}, d_{\{1, \ldots, L\}}, \tilde{\sigma}_{1}^{2}, \ldots, \tilde{\sigma}_{L-1}^{2}\right) \\
&=\max _{\sigma_{j}^{2} \in\left[0, \sigma_{X}^{2}\right], j=1, \ldots, L-1} \psi\left(d_{\{1\}}, \ldots,\right. \\
& d_{\{L\}}, \tilde{d}_{\{1,2\}}, \ldots, \tilde{d}_{\{1, \ldots, L-1\}}, \\
&\left.d_{\{1, \ldots, L\}}, \sigma_{1}^{2}, \ldots, \sigma_{L-1}^{2}\right) \\
& \geq \psi\left(d_{\{1\}}, \ldots, d_{\{L\}}, \tilde{d}_{\{1,2\}}, \ldots, \tilde{d}_{\{1, \ldots, L-1\}},\right. \\
& d_{\{1, \ldots, L\}}, \tilde{\sigma}_{1}^{2}, \ldots, \tilde{\sigma}_{i^{*}-2}^{2}, \sigma_{i^{*}-1}^{2}\left(d_{\left\{1, \ldots, i^{*}\right\}}^{*}\right) \\
&\left.\sigma_{i^{*}}^{2}\left(d_{\left\{1, \ldots, i^{*}\right\}}^{*}\right), \tilde{\sigma}_{i^{*}+1}^{2}, \ldots, \tilde{\sigma}_{L-1}^{2}\right) \\
& \geq d_{\left\{1, \ldots, i^{*}\right\}} \in\left[0, d_{\left\{1, \ldots, i^{*}\right\}}\right] \psi\left(d_{\{1\}}, \ldots, d_{\{L\}}, \tilde{d}_{\{1,2\}}, \ldots,\right. \\
& \tilde{d}_{\left\{1, \ldots, i^{*}-1\right\}}, d_{\left\{1, \ldots, i^{*}\right\}}, \tilde{d}_{\left\{1, \ldots, i^{*}+1\right\}}, \ldots, \tilde{d}_{\{1, \ldots, L-1\}}, \\
& d_{\{1, \ldots, L\}}, \tilde{\sigma}_{1}^{2}, \ldots, \tilde{\sigma}_{i^{*}-2}^{2}, \sigma_{i^{*}-1}^{2}\left(d_{\left\{1, \ldots, i^{*}\right\}}^{*}\right) \\
&\left.\sigma_{i^{*}}^{2}\left(d_{\left\{1, \ldots, i^{*}\right\}}^{*}\right), \tilde{\sigma}_{i^{*}+1}^{2}, \ldots, \tilde{\sigma}_{L-1}^{2}\right) \\
&= \psi\left(d_{\{1\}}, \ldots, d_{\{L\}}, \tilde{d}_{\{1,2\}}, \ldots, \tilde{d}_{\left\{1, \ldots, i^{*}-1\right\}},\right. \\
& d_{\left\{1, \ldots, i^{*}\right\}}^{*}, \tilde{d}_{\left\{1, \ldots, i^{*}+1\right\}}, \ldots, \tilde{d}_{\{1, \ldots, L-1\}}, d_{\{1, \ldots, L\}}, \\
& \tilde{\sigma}_{1}^{2}, \ldots, \tilde{\sigma}_{i^{*}-2}^{2}, \sigma_{i^{*}-1}^{2}\left(d_{\left\{1, \ldots, i^{*}\right\}}^{*}\right), \\
&\left.\sigma_{i^{*}}^{2}\left(d_{\left\{1, \ldots, i^{*}\right\}}^{*}\right), \tilde{\sigma}_{i^{*}+1}^{2}, \ldots, \tilde{\sigma}_{L-1}^{2}\right) \\
&= \sigma_{j}^{2} \in\left[0, \sigma_{X}^{2}\right], j=1, \ldots, L-1 \\
& \tilde{d}_{\{1,2\}}, \ldots, \tilde{d}_{\left\{1, \ldots, i^{*}-1\right\}}, d_{\left\{1, \ldots, i^{*}\right\}}, \\
&\left.\tilde{d}_{\left\{1, \ldots, i^{*}+1\right\}}, \ldots, \tilde{d}_{\{1, \ldots, L-1\}}, d_{\{1, \ldots, L\}}, \sigma_{1}^{2}, \ldots, \sigma_{L-1}^{2}\right) .
\end{aligned}
$$

Since $\left(\tilde{d}_{\{1,2\}}, \ldots, \tilde{d}_{\{1, \ldots, L-1\}}, \tilde{\sigma}_{1}^{2}, \ldots, \tilde{\sigma}_{L-1}^{2}\right)$ is a solution to the min-max game in (4), the inequalities in (36) and (37) must be equalities. Note that the equality holds in (36) if and only if $\sigma_{i^{*}-1}^{2}\left(d_{\left\{1, \ldots, i^{*}\right\}}^{*}\right)=\tilde{\sigma}_{i^{*}-1}^{2}$ and $\sigma_{i^{*}}^{2}\left(d_{\left\{1, \ldots, i^{*}\right\}}^{*}\right)=\tilde{\sigma}_{i^{*}}^{2}$ while the equality holds in (37) if and only if one of the following three conditions is satisfied:

1) $d_{\left\{1, \ldots, i^{*}\right\}}^{*}=\tilde{d}_{\left\{1, \ldots, i^{*}\right\}}$

2) $\sigma_{i^{*}-1}^{2}\left(d_{\left\{1, \ldots, i^{*}\right\}}^{*}\right)=\sigma_{i^{*}}^{2}\left(d_{\left\{1, \ldots, i^{*}\right\}}^{*}\right)=0$;

3) $\alpha_{i^{*}}=\alpha_{i^{*}+1}$ and $\sigma_{i^{*}-1}^{2}\left(d_{\left\{1, \ldots, i^{*}\right\}}^{*}\right)=\sigma_{i^{*}}^{2}\left(d_{\left\{1, \ldots, i^{*}\right\}}^{*}\right)$.
Therefore, $\tilde{d}_{\left\{1, \ldots, i^{*}\right\}}, \quad \tilde{\sigma}_{i^{*}-1}^{2}$, and $\tilde{\sigma}_{i^{*}}^{2}$ satisfy (34) [or (35)], which leads a contradiction with the assumption that (34) [or (35)] is violated. As a consequence, $\left(\tilde{d}_{\{1,2\}}, \ldots, \tilde{d}_{\{1, \ldots, L-1\}}, \tilde{\sigma}_{1}^{2}, \ldots, \tilde{\sigma}_{L-1}^{2}\right)$ must be a saddle point.

Now we proceed to treat the case where some of the distortion constraints are equal to $\sigma_{X}^{2}$ using a limiting argument. We subtract each distortion constraint by $\epsilon$. It is clear that the resulting distortion constraints are all less than $\sigma_{X}^{2}$; therefore, the aforedescribed argument can be used to prove the existence of a saddle point. By sending $\epsilon$ to zero, one can find a converging subsequence of saddle points. It can be readily verified that the limiting value is a saddle point of the original max-min/min-max game. This completes the proof of Theorem 3 .

A detailed characterization of the saddle point can be found in Appendix E.

\section{CONCLUSION}

We have characterized the rate region of Gaussian multiple description coding with individual and central distortion constraints. Different from the 2-description case and the minimum sum-rate case where a single auxiliary random vector suffices to yield a matching converse, multiple auxiliary random vectors $Z_{1}^{n}, \ldots, Z_{L-1}^{n}$ are needed in the current setting. Note that although it is not necessary to specify the joint distribution of $Z_{1}^{n}, \ldots, Z_{L-1}^{n}$ in our proof, one may put them into the same probability space and create a certain structure. In particular, if only individual and central distortion constraints are imposed, the auxiliary random vectors $Z_{1}^{n}, \ldots, Z_{L-1}^{n}$ can be arranged to form a Markov chain with an order determined by $\alpha_{1}, \ldots, \alpha_{L}$; however, this order can be affected if additional distortion constraints are introduced. It is worth noting that Markov structured auxiliary random vectors have also been used in a recent work [12] to derive an outer bound of the Gaussian multiple description problem with symmetric distortion constraints that is within a constant gap from the inner bounds. Our result indicates that in the case where only individual and central distortion constraints are imposed, one can in fact obtain the true rate region by systematically optimizing the auxiliary random vectors.

\section{APPENDIX A \\ PROOF OF LEMMA 1}

Consider the following two cases.

1) Case 1: $\sigma_{2}^{2}>\sigma_{1}^{2}$. We can write $N_{2}^{n}=N_{1}^{n}+\tilde{N}^{n}$, where $\tilde{N}^{n}$ is an $n$-dimensional Gaussian random vector with covariance matrix $\left(\sigma_{2}^{2}-\sigma_{1}^{2}\right) I_{n}$, and $\tilde{N}^{n}$ is independent of $N_{1}^{n}$. Note that

$$
\begin{aligned}
\mu_{1} h\left(S^{n}+N_{1}^{n} \mid T\right)-\mu_{2} h\left(S^{n}+N_{2}^{n} \mid T\right) \\
+\left(\mu_{2}-\mu_{1}\right) h\left(S^{n} \mid T\right) \\
=\mu_{1} h\left(S^{n}+N_{1}^{n} \mid T\right) \\
\quad-\mu_{1} h\left(S^{n}+N_{1}^{n}+\tilde{N}^{n} \mid T\right) \\
\quad-\left(\mu_{2}-\mu_{1}\right) h\left(S^{n}+N_{2}^{n} \mid T\right) \\
\quad+\left(\mu_{2}-\mu_{1}\right) h\left(S^{n} \mid T\right) \\
=-\mu_{1} I\left(\tilde{N}^{n} ; S^{n}+N_{1}^{n}+\tilde{N}^{n} \mid T\right) \\
\quad-\left(\mu_{2}-\mu_{1}\right) I\left(N_{2}^{n} ; S^{n}+N_{2}^{n} \mid T\right) .
\end{aligned}
$$


Define $\hat{S}^{n}=\mathbb{E}\left(S^{n} \mid T\right)$. We have

$$
\begin{aligned}
I( & \left.\tilde{N}^{n} ; S^{n}+N_{1}^{n}+\tilde{N}^{n} \mid T\right) \\
= & h\left(\tilde{N}^{n}\right)-h\left(\tilde{N}^{n} \mid S^{n}+N_{1}^{n}+\tilde{N}^{n}, T\right) \\
\geq & h\left(\tilde{N}^{n}\right)-h\left(\tilde{N}^{n} \mid S^{n}-\hat{S}^{n}+N_{1}^{n}+\tilde{N}^{n}\right) \\
\geq & \sum_{m=1}^{n}(h(\tilde{N}(m))-h(\tilde{N}(m) \mid S(m) \\
& \left.\left.-\hat{S}(m)+N_{1}(m)+\tilde{N}(m)\right)\right) \\
= & \sum_{m=1}^{n} I\left(\tilde{N}(m) ; S(m)-\hat{S}(m)+N_{1}(m)+\tilde{N}(m)\right) \\
\geq & \sum_{m=1}^{n} \frac{1}{2} \log \left(\frac{\mathbb{E}(S(m)-\hat{S}(m))^{2}+\sigma_{2}^{2}}{\mathbb{E}(S(m)-\hat{S}(m))^{2}+\sigma_{1}^{2}}\right) \\
\geq & \frac{n}{2} \log \left(\frac{d^{\prime}+\sigma_{2}^{2}}{d^{\prime}+\sigma_{1}^{2}}\right)
\end{aligned}
$$

where (38) follows from the worst additive noise lemma [13, Lemma II.2], and (39) follows from the convexity and monotonicity of $\log \left(\frac{a+b}{b}\right)$ in $b$ (when $a>0$ and $b \geq 0$ ) as well as the fact that $\operatorname{tr}\left(\operatorname{cov}\left(S^{n} \mid T\right)\right) \leq n d^{\prime}$. Similarly, it can be shown that

$I\left(N_{i}^{n} ; S^{n}+N_{i}^{n} \mid T\right) \geq \frac{n}{2} \log \left(\frac{d^{\prime}+\sigma_{i}^{2}}{d^{\prime}}\right), \quad i=1,2$.

In view of the fact that both $\mu_{1}$ and $\mu_{2}-\mu_{1}$ are nonnegative, we have

$$
\begin{array}{rl}
\mu_{1} & h\left(S^{n}+N_{1}^{n} \mid T\right)-\mu_{2} h\left(S^{n}+N_{2}^{n} \mid T\right) \\
+ & \left(\mu_{2}-\mu_{1}\right) h\left(S^{n} \mid T\right) \\
\leq & \frac{\mu_{1} n}{2} \log \left(\frac{d^{\prime}+\sigma_{1}^{2}}{d^{\prime}+\sigma_{2}^{2}}\right) \\
& +\frac{\left(\mu_{2}-\mu_{1}\right) n}{2} \log \left(\frac{d^{\prime}}{d^{\prime}+\sigma_{2}^{2}}\right) \\
= & \frac{\mu_{1} n}{2} \log \left(d^{\prime}+\sigma_{1}^{2}\right)-\frac{\mu_{2} n}{2} \log \left(d^{\prime}+\sigma_{2}^{2}\right) \\
& +\frac{\left(\mu_{2}-\mu_{1}\right) n}{2} \log \left(d^{\prime}\right) .
\end{array}
$$

2) Case 2: $\sigma_{2}^{2} \leq \sigma_{1}^{2}$. By the conditional version of Costa's entropy power inequality [10] (cf. [14]), we have

$$
\begin{aligned}
h\left(S^{n}+N_{2}^{n} \mid T\right) & \\
= & h\left(S^{n}+\sqrt{\frac{\sigma_{2}^{2}}{\sigma_{1}^{2}}} N_{1}^{n} \mid T\right) \\
\geq & \frac{n}{2} \log \left(\left(1-\frac{\sigma_{2}^{2}}{\sigma_{1}^{2}}\right) \exp \left(\frac{2}{n} h\left(S^{n} \mid T\right)\right)\right. \\
& \left.+\frac{\sigma_{2}^{2}}{\sigma_{1}^{2}} \exp \left(\frac{2}{n} h\left(S^{n}+N_{1}^{n} \mid T\right)\right)\right) .
\end{aligned}
$$

Therefore

$$
\begin{aligned}
& \mu_{1} h\left(S^{n}+N_{1}^{n} \mid T\right)-\mu_{2} h\left(S^{n}+N_{2}^{n} \mid T\right) \\
&+\left(\mu_{2}-\mu_{1}\right) h\left(S^{n} \mid T\right) \\
& \leq \mu_{1} h\left(S^{n}+N_{1}^{n} \mid T\right)-\frac{\mu_{2} n}{2} \\
& \quad \times \log \left(\left(1-\frac{\sigma_{2}^{2}}{\sigma_{1}^{2}}\right) \exp \left(\frac{2}{n} h\left(S^{n} \mid T\right)\right)\right. \\
&\left.+\frac{\sigma_{2}^{2}}{\sigma_{1}^{2}} \exp \left(\frac{2}{n} h\left(S^{n}+N_{1}^{n} \mid T\right)\right)\right) \\
&+\left(\mu_{2}-\mu_{1}\right) h\left(S^{n} \mid T\right) \\
&= \mu_{1} I\left(N_{1}^{n} ; S^{n}+N_{1}^{n} \mid T\right) \\
&-\frac{\mu_{2} n}{2} \log \left(1-\frac{\sigma_{2}^{2}}{\sigma_{1}^{2}}+\frac{\sigma_{2}^{2}}{\sigma_{1}^{2}}\right. \\
&\left.\times \exp \left(\frac{2}{n} I\left(N_{1}^{n} ; S^{n}+N_{1}^{n} \mid T\right)\right)\right) .
\end{aligned}
$$

Now it follows from (40) that

$$
\begin{aligned}
\mu_{1} h( & \left.S^{n}+N_{1}^{n} \mid T\right)-\mu_{2} h\left(S^{n}+N_{2}^{n} \mid T\right) \\
+ & \left(\mu_{2}-\mu_{1}\right) h\left(S^{n} \mid T\right) \\
\leq & \max _{d \in\left[0, d^{\prime}\right]} \frac{\mu_{1} n}{2} \log \left(\frac{d+\sigma_{1}^{2}}{d}\right) \\
& -\frac{\mu_{2} n}{2} \log \left(1-\frac{\sigma_{2}^{2}}{\sigma_{1}^{2}}+\frac{\left(d+\sigma_{1}^{2}\right) \sigma_{2}^{2}}{d \sigma_{1}^{2}}\right) \\
= & \max _{d \in\left[0, d^{\prime}\right]} \frac{\mu_{1} n}{2} \log \left(d+\sigma_{1}^{2}\right) \\
& -\frac{\mu_{2} n}{2} \log \left(d+\sigma_{2}^{2}\right)+\frac{\left(\mu_{2}-\mu_{1}\right) n}{2} \log (d) .
\end{aligned}
$$

The proof is complete.

\section{APPENDIX B}

PROOF OF LEMMA 2

We shall only treat the case $\max \left\{d_{\{1, \ldots, j\}}, d_{\{j+1\}}\right\}<\sigma_{X}^{2}$ since the other one is straightforward to verify. Consider the objective function $G_{j}\left(\sigma_{j}^{2}\right)$ shown in the equation at the bottom of the page. It is easy to verify that

$$
\begin{aligned}
\frac{\partial G_{j}\left(\sigma_{j}^{2}\right)}{\partial \sigma_{j}^{2}}= & \left(\frac{\sigma_{X}^{2} d_{\{1, \ldots, j+1\}}}{\sigma_{X}^{2}-d_{\{1, \ldots, j+1\}}}+\sigma_{j}^{2}\right)^{-1} \\
& -\left(\frac{\sigma_{X}^{2} d_{\{1, \ldots, j\}}}{\sigma_{X}^{2}-d_{\{1, \ldots, j\}}}+\sigma_{j}^{2}\right)^{-1} \\
& -\left(\frac{\sigma_{X}^{2} d_{\{j+1\}}}{\sigma_{X}^{2}-d_{\{j+1\}}}+\sigma_{j}^{2}\right)^{-1} \\
\frac{\partial^{2} G_{j}\left(\sigma_{j}^{2}\right)}{\partial\left(\sigma_{j}^{2}\right)^{2}=} & -\left(\frac{\sigma_{X}^{2} d_{\{1, \ldots, j+1\}}}{\sigma_{X}^{2}-d_{\{1, \ldots, j+1\}}}+\sigma_{j}^{2}\right)^{-2} \\
& +\left(\frac{\sigma_{X}^{2} d_{\{1, \ldots, j\}}}{\sigma_{X}^{2}-d_{\{1, \ldots, j\}}}+\sigma_{j}^{2}\right)^{-2}
\end{aligned}
$$

$$
G_{j}\left(\sigma_{j}^{2}\right)=\log \left(\frac{\sigma_{X}^{4}\left(\sigma_{X}^{2} d_{\{1, \ldots, j+1\}}-d_{\{1, \ldots, j+1\}} \sigma_{j}^{2}+\sigma_{X}^{2} \sigma_{j}^{2}\right)}{\left(\sigma_{X}^{2} d_{\{1, \ldots, j\}}-d_{\{1, \ldots, j\}} \sigma_{j}^{2}+\sigma_{X}^{2} \sigma_{j}^{2}\right)\left(\sigma_{X}^{2} d_{\{j+1\}}-d_{\{j+1\}} \sigma_{j}^{2}+\sigma_{X}^{2} \sigma_{j}^{2}\right)}\right) .
$$




$$
+\left(\frac{\sigma_{X}^{2} d_{\{j+1\}}}{\sigma_{X}^{2}-d_{\{j+1\}}}+\sigma_{j}^{2}\right)^{-2} .
$$

For any $\hat{\sigma}_{j}^{2} \in\left[0, \sigma_{X}^{2}\right]$ such that $\left.\frac{\partial G_{j}\left(\sigma_{j}^{2}\right)}{\partial \sigma_{j}^{2}}\right|_{\sigma_{j}^{2}=\hat{\sigma}_{j}^{2}}=0$, we have

$$
\begin{aligned}
\left.\frac{\partial^{2} G_{j}\left(\sigma_{j}^{2}\right)}{\partial\left(\sigma_{j}^{2}\right)^{2}}\right|_{\sigma_{j}^{2}=\hat{\sigma}_{j}^{2}} \\
<-\left(\frac{\sigma_{X}^{2} d_{\{1, \ldots, j+1\}}}{\sigma_{X}^{2}-d_{\{1, \ldots, j+1\}}}+\hat{\sigma}_{j}^{2}\right)^{-2} \\
\quad+\left(\left(\frac{\sigma_{X}^{2} d_{\{1, \ldots, j\}}}{\sigma_{X}^{2}-d_{\{1, \ldots, j\}}}+\hat{\sigma}_{j}^{2}\right)^{-1}\right. \\
\left.\quad+\left(\frac{\sigma_{X}^{2} d_{\{j+1\}}}{\sigma_{X}^{2}-d_{\{j+1\}}}+\hat{\sigma}_{j}^{2}\right)^{-1}\right)^{2}
\end{aligned}
$$$$
=0 \text {. }
$$

Therefore, $G_{j}\left(\hat{\sigma}_{j}^{2}\right)$ is a local maximum for any $\hat{\sigma}_{j}^{2} \in\left[0, \sigma_{X}^{2}\right]$ satisfying $\left.\frac{\partial G_{j}\left(\sigma_{j}^{2}\right)}{\partial \sigma_{j}^{2}}\right|_{\sigma_{j}^{2}=\hat{\sigma}_{j}^{2}}=0$, and there is no local minimum in $\left(0, \sigma_{X}^{2}\right)$.

Note that

$$
\begin{aligned}
& \left.\frac{\partial G_{j}\left(\sigma_{j}^{2}\right)}{\partial \sigma_{j}^{2}}\right|_{\sigma_{j}^{2}=0} \\
& \quad=\frac{1}{d_{\{1, \ldots, j+1\}}}+\frac{1}{\sigma_{X}^{2}}-\frac{1}{d_{\{1, \ldots, j\}}}-\frac{1}{d_{\{j+1\}}} \\
& \left.\frac{\partial G_{j}\left(\sigma_{j}^{2}\right)}{\partial \sigma_{j}^{2}}\right|_{\sigma_{j}^{2}=\sigma_{X}^{2}} \\
& =\frac{1}{\sigma_{X}^{4}}\left(d_{\{1, \ldots, j\}}+d_{\{j+1\}}-d_{\{1, \ldots, j+1\}}-\sigma_{X}^{2}\right) .
\end{aligned}
$$

It is easy to show that

$$
\left(\frac{1}{d_{\{1, \ldots, j\}}}+\frac{1}{d_{\{j+1\}}}-\frac{1}{\sigma_{X}^{2}}\right)^{-1} \geq d_{\{1, \ldots, j\}}+d_{\{j+1\}}-\sigma_{X}^{2}
$$

with inequality if and only if $\max \left\{d_{\{1, \ldots, j\}}, d_{\{j+1\}}\right\}=\sigma_{X}^{2}$. Therefore, we have

$$
\begin{gathered}
\left.\frac{\partial G_{j}\left(\sigma_{j}^{2}\right)}{\partial \sigma_{j}^{2}}\right|_{\sigma_{j}^{2}=0} \leq\left. 0 \Rightarrow \frac{\partial G_{j}\left(\sigma_{j}^{2}\right)}{\partial \sigma_{j}^{2}}\right|_{\sigma_{j}^{2}=\sigma_{X}^{2}} \leq 0, \\
\left.\frac{\partial G_{j}\left(\sigma_{j}^{2}\right)}{\partial \sigma_{j}^{2}}\right|_{\sigma_{j}^{2}=\sigma_{X}^{2}} \geq\left. 0 \Rightarrow \frac{\partial G_{j}\left(\sigma_{j}^{2}\right)}{\partial \sigma_{j}^{2}}\right|_{\sigma_{j}^{2}=0} \geq 0 .
\end{gathered}
$$

In both (41) and (42), if the first inequality is strict, then the second inequality is also strict. Furthermore, $\left.\frac{\partial G_{j}\left(\sigma_{j}^{2}\right)}{\partial \sigma_{j}^{2}}\right|_{\sigma_{j}^{2}=0} \leq 0$ and $\left.\frac{\partial G_{j}\left(\sigma_{j}^{2}\right)}{\partial \sigma_{j}^{2}}\right|_{\sigma_{j}^{2}=\sigma_{X}^{2}} \geq 0$ hold simultaneously if and only if one of $d_{\{1, \ldots, j\}}$ and $d_{\{j+1\}}$ is equal to $d_{\{1, \ldots, j+1\}}$ and the other is equal to $\sigma_{X}^{2}$ (in which case $G_{j}\left(\sigma_{j}^{2}\right)$ does not depend on $\sigma_{j}^{2}$ ). Since both $d_{\{1, \ldots, j\}}$ and $d_{\{j+1\}}$ are less than $\sigma_{X}^{2}$ under our assumption, only the following three cases need to be considered.

1) Case 1: $\left.\frac{\partial G_{j}\left(\sigma_{j}^{2}\right)}{\partial \sigma_{j}^{2}}\right|_{\sigma_{j}^{2}=0} \leq 0$ and $\left.\frac{\partial G_{j}\left(\sigma_{j}^{2}\right)}{\partial \sigma_{j}^{2}}\right|_{\sigma_{j}^{2}=\sigma_{X}^{2}}<0$. We have $\frac{\partial G_{j}\left(\sigma_{j}^{2}\right)}{\partial \sigma_{j}^{2}}<0$ for $\sigma_{j}^{2} \in\left(0, \sigma_{X}^{2}\right]$. Otherwise, if $\frac{\partial G_{j}\left(\sigma_{j}^{2}\right)}{\partial \sigma_{j}^{2}}=0$ for some $\sigma_{j}^{2} \in\left(0, \sigma_{X}^{2}\right)$, then $G_{j}\left(\sigma_{j}^{2}\right)$ is a local maximum, and there must exist a local minimum in $\left(0, \sigma_{j}^{2}\right)$, which leads to a contradiction with the fact that no local minimum exists in $\left(0, \sigma_{X}^{2}\right)$. Therefore, $G_{j}\left(\sigma_{j}^{2}\right)$ is a strictly decreasing function of $\sigma_{j}^{2}$ from 0 to $\sigma_{X}^{2}$.

2) Case 2: $\left.\frac{\partial G_{j}\left(\sigma_{j}^{2}\right)}{\partial \sigma_{j}^{2}}\right|_{\sigma_{j}^{2}=\sigma_{X}^{2}} \geq 0$ and $\left.\frac{\partial G_{j}\left(\sigma_{j}^{2}\right)}{\partial \sigma_{j}^{2}}\right|_{\sigma_{j}^{2}=0}>0$. We have $\frac{\partial G_{j}\left(\sigma_{j}^{2}\right)}{\partial \sigma_{j}^{2}}>0$ for $\sigma_{j}^{2} \in\left[0, \sigma_{X}^{2}\right)$. Otherwise, if $\frac{\partial G_{j}\left(\sigma_{j}^{2}\right)}{\partial \sigma_{j}^{2}}=0$ for some $\sigma_{j}^{2} \in\left(0, \sigma_{X}^{2}\right)$, then $G_{j}\left(\sigma_{j}^{2}\right)$ is a local maximum, and there must exist a local minimum in $\left(\sigma_{j}^{2}, \sigma_{X}^{2}\right)$, which leads to a contradiction. Therefore, $G_{j}\left(\sigma_{j}^{2}\right)$ is a strictly increasing function of $\sigma_{j}^{2}$ from 0 to $\sigma_{X}^{2}$.

3) Case 3: $\left.\frac{\partial G_{j}\left(\sigma_{j}^{2}\right)}{\partial \sigma_{j}^{2}}\right|_{\sigma_{j}^{2}=0}>0$ and $\left.\frac{\partial G_{j}\left(\sigma_{j}^{2}\right)}{\partial \sigma_{j}^{2}}\right|_{\sigma_{j}^{2}=\sigma_{X}^{2}}<0$. There exists a unique $\hat{\sigma}_{j}^{2} \in\left(0, \sigma_{X}^{2}\right)$ satisfying $\left.\frac{\partial G_{j}\left(\sigma_{j}^{2}\right)}{\partial \sigma_{j}^{2}}\right|_{\sigma_{j}^{2}=\hat{\sigma}_{j}^{2}}=$ 0 . We have $\frac{\partial G_{j}\left(\sigma_{j}^{2}\right)}{\partial \sigma_{2}^{2}}>0$ for $\sigma_{j}^{2} \in\left[0, \hat{\sigma}_{j}^{2}\right)$, and $\frac{\partial G_{j}\left(\sigma_{j}^{2}\right)}{\partial \sigma^{2}}<$ 0 for $\sigma_{j}^{2} \in\left(\hat{\sigma}_{j}^{2}, \sigma_{X}^{2}\right]$. The existence of such a $\hat{\sigma}_{j}^{2}$ follows from the intermediate value theorem. To prove the uniqueness, we assume that there exists a $\bar{\sigma}_{j}^{2} \neq \hat{\sigma}_{j}^{2}$ such that $\left.\frac{\partial G_{j}\left(\sigma_{j}^{2}\right)}{\partial \sigma_{j}^{2}}\right|_{\sigma_{j}^{2}=\bar{\sigma}_{j}^{2}}=0$. Since both $G_{j}\left(\hat{\sigma}_{j}^{2}\right)$ and $G_{j}\left(\bar{\sigma}_{j}^{2}\right)$ are local maxima, there must exist a local minimum between $\hat{\sigma}_{j}^{2}$ and $\bar{\sigma}_{j}^{2}$, which leads to a contradiction. Therefore, $G_{j}\left(\sigma_{j}^{2}\right)$ is a strictly increasing function of $\sigma_{j}^{2}$ from 0 to $\hat{\sigma}_{j}^{2}$ and a strictly decreasing function of $\sigma_{j}^{2}$ from $\hat{\sigma}_{j}^{2}$ to $\sigma_{X}^{2}$.

Now we proceed to show that the maximizers $\sigma_{j}^{2}$, $j=1, \ldots, L-1$, given in (19) are monotonically decreasing continuous functions of $d_{\{1, \ldots, j+1\}}$ and monotonically increasing continuous functions of $d_{\{1, \ldots, j\}}$; furthermore, the monotonicity is strict when $\sigma_{j}^{2} \in\left(0, \sigma_{X}^{2}\right)$. We shall focus on the claim regarding $d_{\{1, \ldots, j+1\}}$ since the other one can be proved in a similar manner. Note that for $d_{\{1, \ldots, j+1\}} \in\left(d_{\{1, \ldots, j+1\}}^{\star}, d_{\{1, \ldots, j+1\}}^{\diamond}\right)$, we have $\sigma_{j}^{2}=\hat{\sigma}_{j}^{2} \in\left(0, \sigma_{X}^{2}\right)$. To stress its dependence on $d_{\{1, \ldots, j+1\}}$, we denote $\hat{\sigma}_{j}^{2}$ as $\hat{\sigma}_{j}^{2}\left(d_{\{1, \ldots, j+1\}}\right)$. It is easy to see that

$$
\begin{gathered}
\left(\frac{\sigma_{X}^{2}\left(d_{\{1, \ldots, j+1\}}+\delta\right)}{\sigma_{X}^{2}-\left(d_{\{1, \ldots, j+1\}}+\delta\right)}+\hat{\sigma}_{j}^{2}\left(d_{\{1, \ldots, j+1\}}\right)\right)^{-1} \\
-\left(\frac{\sigma_{X}^{2} d_{\{1, \ldots, j\}}}{\sigma_{X}^{2}-d_{\{1, \ldots, j\}}}+\hat{\sigma}_{j}^{2}\left(d_{\{1, \ldots, j+1\}}\right)\right)^{-1} \\
-\left(\frac{\sigma_{X}^{2} d_{\{j+1\}}}{\sigma_{X}^{2}-d_{\{j+1\}}}+\hat{\sigma}_{j}^{2}\left(d_{\{1, \ldots, j+1\}}\right)\right)^{-1}<0
\end{gathered}
$$

where $\delta$ is a small positive number. Now by the analysis for case 3 , we must have $\hat{\sigma}_{j}^{2}\left(d_{\{1, \ldots, j+1\}}\right)>\hat{\sigma}_{j}^{2}\left(d_{\{1, \ldots, j+1\}}+\delta\right)$. Therefore, $\hat{\sigma}_{j}^{2}\left(d_{\{1, \ldots, j+1\}}\right)$ is a strictly monotonically decreasing function of $d_{\{1, \ldots, j+1\}}$.

We shall prove that $\hat{\sigma}_{j}^{2}\left(d_{\{1, \ldots, j+1\}}\right)$ is a continuous function of $d_{\{1, \ldots, j+1\}}$ by contradiction. Without loss of generality, we assume

$$
\sigma^{2} \triangleq \lim _{d_{\{1, \ldots, j+1\}} \uparrow \bar{d}_{\{1, \ldots, j+1\}}} \hat{\sigma}_{j}^{2}\left(d_{\{1, \ldots, j+1\}}\right) \neq \hat{\sigma}_{j}^{2}\left(\bar{d}_{\{1, \ldots, j+1\}}\right)
$$


for some $\bar{d}_{\{1, \ldots, j+1\}}$. Note that

$$
\begin{aligned}
& \left(\frac{\sigma_{X}^{2} \bar{d}_{\{1, \ldots, j+1\}}}{\sigma_{X}^{2}-\bar{d}_{\{1, \ldots, j+1\}}}+\sigma^{2}\right)^{-1} \\
& -\left(\frac{\sigma_{X}^{2} d_{\{1, \ldots, j\}}}{\sigma_{X}^{2}-d_{\{1, \ldots, j\}}}+\sigma^{2}\right)^{-1} \\
& -\left(\frac{\sigma_{X}^{2} d_{\{j+1\}}}{\sigma_{X}^{2}-d_{\{j+1\}}}+\sigma^{2}\right)^{-1} \\
& =d_{\{1, \ldots, j+1\}} \bar{d}_{\{1, \ldots, j+1\}}\left(\frac{\sigma_{X}^{2} d_{\{1, \ldots, j+1\}}}{\sigma_{X}^{2}-d_{\{1, \ldots, j+1\}}}\right. \\
& \left.\quad+\hat{\sigma}_{j}^{2}\left(d_{\{1, \ldots, j+1\}}\right)\right)^{-1} \\
& \quad-\left(\frac{\sigma_{X}^{2} d_{\{1, \ldots, j\}}}{\sigma_{X}^{2}-d_{\{1, \ldots, j\}}}+\hat{\sigma}_{j}^{2}\left(d_{\{1, \ldots, j+1\}}\right)\right)^{-1} \\
& \quad-\left(\frac{\sigma_{X}^{2} d_{\{j+1\}}}{\sigma_{X}^{2}-d_{\{j+1\}}}+\hat{\sigma}_{j}^{2}\left(d_{\{1, \ldots, j+1\}}\right)\right)^{-1} \\
& =0
\end{aligned}
$$

which is contradictory with the analysis for case 3 .

By leveraging the analysis for case 1 and case 2, one can use a similar argument to show that $\hat{\sigma}_{j}^{2}\left(d_{\{1, \ldots, j+1\}}\right) \downarrow 0$ as $d_{\{1, \ldots, j+1\}} \uparrow d_{\{1, \ldots, j+1\}}^{\diamond}$ and $\hat{\sigma}_{j}^{2}\left(d_{\{1, \ldots, j+1\}}\right) \uparrow \sigma_{X}^{2}$ as $d_{\{1, \ldots, j+1\}} \downarrow d_{\{1, \ldots, j+1\}}^{\star}$ (if $d_{\{1, \ldots, j+1\}}^{\star} \geq 0$ ). The proof is complete.

\section{APPENDIX C}

\section{PROOF OF LEMMA 3}

Consider the following procedure.

1) Initialization: $j=1$ and $\tilde{d}_{\{1\}}=d_{\{1\}}$.

2) Let $\sigma_{j}^{2}$ be a maximizer to the optimization problem shown at the bottom of the page. If $\sigma_{j}^{2}=0$, then set $\tilde{d}_{\{j+1\}}=$ $d_{\{j+1\}}$ and $\tilde{d}_{\{1, \ldots, j+1\}}=\left(\frac{1}{\tilde{d}_{\{1, \ldots, j\}}}+\frac{1}{d_{\{j+1\}}}-\frac{1}{\sigma_{X}^{2}}\right)^{-1}$; if $\sigma_{j}^{2}=\sigma_{X}^{2}$, then set

$$
\tilde{d}_{\{j+1\}}=d_{\{1, \ldots, j+1\}}+\sigma_{X}^{2}-\tilde{d}_{\{1, \ldots, j\}}
$$

and

$$
\tilde{d}_{\{1, \ldots, j+1\}}=d_{\{1, \ldots, j+1\}}
$$

otherwise set

$$
\tilde{d}_{\{j+1\}}=d_{\{j+1\}}
$$

and

$$
\tilde{d}_{\{1, \ldots, j+1\}}=d_{\{1, \ldots, j+1\}} \cdot
$$

3) If $j=L-1$, then stop; otherwise increase $j$ by one and go to 2).

It can be verified that the resulting $\tilde{d}_{\{i\}}, i=1, \ldots, L$, and $\tilde{d}_{\{1, \ldots, i\}}, i=2, \ldots, L$, have the desired property.

\section{APPENDIX D}

PROOF OF LEMMA 5

Consider the objective function

$$
\begin{aligned}
Q\left(d_{\{1, \ldots, i\}}\right) \\
=\frac{\alpha_{i}}{2} \log \left(\sigma_{X}^{2} d_{\{1, \ldots, i\}}-d_{\{1, \ldots, i\}} \sigma_{i-1}^{2}+\sigma_{X}^{2} \sigma_{i-1}^{2}\right) \\
\quad-\frac{\alpha_{i}-\alpha_{i+1}}{2} \log \left(d_{\{1, \ldots, i\}}\right) \\
\quad-\frac{\alpha_{i+1}}{2} \log \left(\sigma_{X}^{2} d_{\{1, \ldots, i\}}-d_{\{1, \ldots, i\}} \sigma_{i}^{2}+\sigma_{X}^{2} \sigma_{i}^{2}\right) .
\end{aligned}
$$

One can easily verify the second equation at the bottom of the page.

1) Case 1: $\alpha_{i}>\alpha_{i+1}$ and $\alpha_{i+1} \sigma_{i}^{2}\left(\sigma_{X}^{2}-\sigma_{i-1}^{2}\right)<$ $\alpha_{i} \sigma_{i-1}^{2}\left(\sigma_{X}^{2}-\sigma_{i}^{2}\right)$. We have $\frac{\partial Q\left(d_{\{1, \ldots, i\}}\right)}{\partial d_{\{1, \ldots, i\}}}<0$ for all $d_{\{1, \ldots, i\}} \in\left(0, d_{\{1, \ldots, i\}}^{\prime}\right]$. Therefore, the minimum is attained at $d_{\{1, \ldots, i\}} \stackrel{d_{\{1, \ldots, i\}}^{\prime}}{=}$

2) Case 2: $\alpha_{i}>\alpha_{i+1}$ and $\alpha_{i+1} \sigma_{i}^{2}\left(\sigma_{X}^{2}-\sigma_{i-1}^{2}\right)>$ $\alpha_{i} \sigma_{i-1}^{2}\left(\sigma_{X}^{2}-\sigma_{i}^{2}\right)$. We have $\frac{\partial Q\left(d_{\{1, \ldots, i\}}\right)}{\partial d_{\{1, \ldots, i\}}}<0$ for $d_{\{1, \ldots, i\}} \in\left(0, \hat{d}_{\{1, \ldots, i\}}\right)$ and $\frac{\partial Q\left(d_{\{1, \ldots, i\}}\right)}{\partial d_{\{1, \ldots, i\}}}>0$ for $d_{\{1, \ldots, i\}}>\hat{d}_{\{1, \ldots, i\}}$, where

$$
\hat{d}_{\{1, \ldots, i\}}=\frac{\left(\alpha_{i}-\alpha_{i+1}\right) \sigma_{X}^{2} \sigma_{i-1}^{2} \sigma_{i}^{2}}{\alpha_{i+1} \sigma_{i}^{2}\left(\sigma_{X}^{2}-\sigma_{i-1}^{2}\right)-\alpha_{i} \sigma_{i-1}^{2}\left(\sigma_{X}^{2}-\sigma_{i}^{2}\right)} .
$$

Therefore, the minimum is attained at $d_{\{1, \ldots, i\}}=$ $\min \left\{\hat{d}_{\{1, \ldots, i\}}, d_{\{1, \ldots, i\}}^{\prime}\right\}$.

3) Case 3: $\alpha_{i}>\alpha_{i+1}, \alpha_{i+1} \sigma_{i}^{2}\left(\sigma_{X}^{2}-\sigma_{i-1}^{2}\right)=\alpha_{i} \sigma_{i-1}^{2}\left(\sigma_{X}^{2}-\right.$ $\left.\sigma_{i}^{2}\right)$ and $\sigma_{i-1}^{2} \sigma_{i}^{2}>0$. We have $\frac{\partial Q\left(d_{\{1, \ldots, i\}}\right)}{\partial d_{\{1, \ldots, i\}}}<0$ for $d_{\{1, \ldots, i\}} \in\left(0, d_{\{1, \ldots, i\}}^{\prime}\right]$. Therefore, the minimum is attained at $d_{\{1, \ldots, i\}}=d_{\{1, \ldots, i\}}^{\prime}$.

4) Case 4: $\sigma_{i-1}^{2}=\sigma_{i}^{2}=0$. The objective function is a constant.

5) Case 5: $\alpha_{i}=\alpha_{i+1}$ and $\sigma_{i-1}^{2}>\sigma_{i}^{2}$. We have $\frac{\partial Q\left(d_{\{1, \ldots, i\}}\right)}{\partial d_{\{1, \ldots, i\}}}<0$ for all $d_{\{1, \ldots, i\}} \in\left(0, d_{\{1, \ldots, i\}}^{\prime}\right]$. Therefore, the minimum is attained at $d_{\{1, \ldots, i\}}=d_{\{1, \ldots, i\}}^{\prime}$.

6) Case 6: $\alpha_{i}=\alpha_{i+1}$ and $\sigma_{i-1}^{2}<\sigma_{i}^{2}$. We have $\frac{\partial Q\left(d_{\{1, \ldots, i\}}\right)}{\partial d_{\{1, \ldots, i\}}}>0$ for all $d_{\{1, \ldots, i\}} \in\left(0, d_{\{1, \ldots, i\}}^{\prime}\right]$. Therefore, the minimum is attained at $d_{\{1, \ldots, i\}}=0$.

7) Case 7: $\alpha_{i}=\alpha_{i+1}$ and $\sigma_{i-1}^{2}=\sigma_{i}^{2}$. The objective function is a constant.

$$
\max _{\sigma_{j}^{2} \in\left[0, \sigma_{X}^{2}\right]} \log \left(\frac{\sigma_{X}^{4}\left(\sigma_{X}^{2} d_{\{1, \ldots, j+1\}}-d_{\{1, \ldots, j+1\}} \sigma_{j}^{2}+\sigma_{X}^{2} \sigma_{j}^{2}\right)}{\left(\sigma_{X}^{2} \tilde{d}_{\{1, \ldots, j\}}-\tilde{d}_{\{1, \ldots, j\}} \sigma_{j}^{2}+\sigma_{X}^{2} \sigma_{j}^{2}\right)\left(\sigma_{X}^{2} d_{\{j+1\}}-d_{\{j+1\}} \sigma_{j}^{2}+\sigma_{X}^{2} \sigma_{j}^{2}\right)}\right) .
$$

$$
\frac{\partial Q\left(d_{\{1, \ldots, i\}}\right)}{\partial d_{\{1, \ldots, i\}}}=\frac{\left(\alpha_{i+1} \sigma_{X}^{2} \sigma_{i}^{2}\left(\sigma_{X}^{2}-\sigma_{i-1}^{2}\right)-\alpha_{i} \sigma_{X}^{2} \sigma_{i-1}^{2}\left(\sigma_{X}^{2}-\sigma_{i}^{2}\right)\right) d_{\{1, \ldots, i\}}-\left(\alpha_{i}-\alpha_{i+1}\right) \sigma_{X}^{4} \sigma_{i-1}^{2} \sigma_{i}^{2}}{2 d_{\{1, \ldots, i\}}\left(\sigma_{X}^{2} d_{\{1, \ldots, i\}}-d_{\{1, \ldots, i\}} \sigma_{i-1}^{2}+\sigma_{X}^{2} \sigma_{i-1}^{2}\right)\left(\sigma_{X}^{2} d_{\{1, \ldots, i\}}-d_{\{1, \ldots, i\}} \sigma_{i}^{2}+\sigma_{X}^{2} \sigma_{i}^{2}\right)}
$$




\section{APPENDIX E \\ Characterization of THE SADDle PoInT}

We shall assume throughout this appendix that all the distortion constraints are less than $\sigma_{X}^{2}$.

The saddle point is in general unique. To see this, consider two saddle points $\left(\bar{d}_{\{1,2\}}, \ldots, \bar{d}_{\{1, \ldots, L-1\}}, \bar{\sigma}_{1}^{2}, \ldots, \bar{\sigma}_{L-1}^{2}\right)$ and $\left(\underline{d}_{\{1,2\}}, \ldots, \underline{d}_{\{1, \ldots, L-1\}}, \underline{\sigma}_{1}^{2}, \ldots, \underline{\sigma}_{L-1}^{2}\right)$. We must have $\bar{\sigma}_{j}^{2}=$ $\underline{\sigma}_{j}^{2} \triangleq \sigma_{j}^{2}, j=1, \ldots, L-1$, since

$$
\begin{aligned}
& \psi\left(\bar{d}_{\{1,2\}}, \ldots, \bar{d}_{\{1, \ldots, L-1\}}, \bar{\sigma}_{1}^{2}, \ldots, \bar{\sigma}_{L-1}^{2}\right) \\
& \quad \geq \psi\left(\bar{d}_{\{1,2\}}, \ldots, \bar{d}_{\{1, \ldots, L-1\}}, \underline{\sigma}_{1}^{2}, \ldots, \underline{\sigma}_{L-1}^{2}\right) \\
& \quad \geq \psi\left(\underline{d}_{\{1,2\}}, \ldots, \underline{d}_{\{1, \ldots, L-1\}}, \underline{\sigma}_{1}^{2}, \ldots, \underline{\sigma}_{L-1}^{2}\right)
\end{aligned}
$$

and the first inequality becomes equality if and only if $\bar{\sigma}_{j}^{2}=\underline{\sigma}_{j}^{2}$, $j=1, \ldots, L-1$. Furthermore, unless $\sigma_{i-1}^{2}=\sigma_{i}^{2}=0$ or $\alpha_{i}=\alpha_{i+1}, \sigma_{i-1}^{2}=\sigma_{i}^{2}$, we must have $\bar{d}_{\{1, \ldots, i\}}=\underline{d}_{\{1, \ldots, i\}}, i=$ $2, \ldots, L-1$. Note that if there exists an $i$ such that $\vec{d}_{\{1, \ldots, i\}} \neq$ $\underline{d}_{\{1, \ldots, i\}}$, we can find an $i^{\prime} \leq i$ such that $\sigma_{i^{\prime}-1}^{2}=\sigma_{i}^{2}, \bar{d}_{\left\{1, \ldots, i^{\prime}\right\}} \neq$ $\underline{d}_{\left\{1, \ldots, i^{\prime}\right\}}$, and $\bar{d}_{\left\{1, \ldots, i^{\prime}-1\right\}}=\underline{d}_{\left\{1, \ldots, i^{\prime}-1\right\}}\left(\right.$ where $\bar{d}_{\left\{1, \ldots, i^{\prime}-1\right\}}=$ $\underline{d}_{\left\{1, \ldots, i^{\prime}-1\right\}} \triangleq d_{\{1\}}$ if $i^{\prime}=2$ ), which implies $\sigma_{i^{\prime}-1}^{2}=0$ or $\sigma_{i^{\prime}-1}^{2}=\sigma_{X}^{2}$ (cf. (19)). Therefore, it is possible to have $\bar{d}_{\{1, \ldots, i\}} \neq \underline{d}_{\{1, \ldots, i\}}$ only when $\sigma_{i-1}^{2}=\sigma_{i}^{2}=0$ or $\alpha_{i}=\alpha_{i+1}$, $\sigma_{i-1}^{2}=\sigma_{i}^{2}=\sigma_{X}^{2}$.

We also have the following results.

Proposition 1: Let $\left(d_{\{1,2\}}, \ldots, d_{\{1, \ldots, L-1\}}, \sigma_{1}^{2}, \ldots, \sigma_{L-1}^{2}\right)$ be a saddle point. We must have $d_{\{1, \ldots, i\}}>0, i=2, \ldots, L-1$.

Proof: If $d_{\{1, \ldots, i\}}=0$, then we must have $\sigma_{i}^{2}=0$ (cf. (19)), which further implies $\sigma_{i-1}^{2}=0$ (cf. (34) and (35)). Moreover, in view of (19) and the fact that $d_{\{i\}}>0$, one can readily show that $d_{\{1, \ldots, i\}}=0$ together with $\sigma_{i-1}^{2}=0$ implies $d_{\{1, \ldots, i-1\}}=0$. Now it follows by induction that $d_{\{1\}}=0$, which results in a contradiction.

Remark: If $d_{\{1, \ldots, i\}}=0$ for some $i$, then we have

$\psi\left(d_{\{1\}}, \ldots, d_{\{L\}}, d_{\{1,2\}}, \ldots, d_{\{1, \ldots, L\}}, \sigma_{1}^{2}, \ldots, \sigma_{L-1}^{2}\right)=\infty$.

On the other hand, it follows by Theorem 3 that

$$
\begin{aligned}
& \psi\left(d_{\{1\}}, \ldots, d_{\{L\}}, d_{\{1,2\}}, \ldots, d_{\{1, \ldots, L\}}, \sigma_{1}^{2}, \ldots, \sigma_{L-1}^{2}\right) \\
& =\kappa\left(d_{\{1\}}, \ldots, d_{\{L\}}, d_{\{1,2\}}^{\prime}, \ldots, d_{\{1, \ldots, L-1\}}^{\prime}, d_{\{1, \ldots, L\}}\right)
\end{aligned}
$$

which is clearly finite. This gives an alternative proof of Proposition 1.

Proposition 2: Let $\left(d_{\{1,2\}}, \ldots, d_{\{1, \ldots, L-1\}}, \sigma_{1}^{2}, \ldots, \sigma_{L-1}^{2}\right)$ be a saddle point. If $\sigma_{i^{*}-1}^{2}=0$ for some $i^{*}$, then $\sigma_{i}^{2}=0$ for all $i \geq i^{*}$.

Proof: If $\sigma_{i^{*}}^{2}>0$, then we have $\alpha_{i^{*}+1} \sigma_{i^{*}}^{2}\left(\sigma_{X}^{2}-\sigma_{i^{*}-1}^{2}\right)>$ $\alpha_{i^{*}} \sigma_{i^{*}-1}^{2}\left(\sigma_{X}^{2}-\sigma_{i^{*}}^{2}\right)$ and $\sigma_{i^{*}}^{2}>\sigma_{i^{*}-1}^{2}$. It follows by (34) and (35) that $d_{\left\{1, \ldots, i^{*}\right\}}=0$, which leads to a contradiction with Proposition 1. Therefore, we must have $\sigma_{i^{*}}^{2}=0$. The proof is complete by induction.

Now we proceed to give a more explicit characterization of the saddle point $\left(d_{\{1,2\}}, \ldots, d_{\{1, \ldots, L-1\}}, \sigma_{1}^{2}, \ldots, \sigma_{L-1}^{2}\right)$ for the case where only individual and central distortion constraints are imposed. We shall set $d_{\{1, \ldots, i\}}^{\prime}=\min _{j \in\{1, \ldots, i\}} d_{\{j\}}, i=$
$2, \ldots, L-1$, to make the auxiliary distortion constraints redundant.

First, one can make the following two observations by inspecting (19), (34), and (35).

1) If $\alpha_{i}>\alpha_{i+1}$ for some $i \geq 2$, then either $\sigma_{i-1}=\sigma_{i}=0$ or $\alpha_{i+1} \sigma_{i}\left(\sigma_{X}^{2}-\sigma_{i-1}^{2}\right)>\alpha_{i} \sigma_{i-1}^{2}\left(\sigma_{X}^{2}-\sigma_{i}^{2}\right)$ (which further implies $\left.\sigma_{i}^{2}>\sigma_{i-1}^{2}\right)$; moreover, if $\alpha_{i+1} \sigma_{i}\left(\sigma_{X}^{2}-\sigma_{i-1}^{2}\right)>$ $\alpha_{i} \sigma_{i-1}^{2}\left(\sigma_{X}^{2}-\sigma_{i}^{2}\right)$, then $d_{\{1, \ldots, i\}}=\hat{d}_{\{1, \ldots, i\}}$.

2) If $\alpha_{i}=\alpha_{i+1}$ for some $i \geq 2$, then $\sigma_{i-1}^{2}=\sigma_{i}^{2}$.

These two observations, together with Proposition 2, lead to the following classification.

1) Case 1: $\sigma_{1}^{2}=\cdots=\sigma_{L-1}^{2}=0$. We must have

$$
\begin{aligned}
d_{\{1, \ldots, i\}} \geq\left(\frac{1}{d_{\{1, \ldots, i-1\}}}+\frac{1}{d_{\{i\}}}-\frac{1}{\sigma_{X}^{2}}\right)^{-1}, \\
i=2, \ldots, L-1
\end{aligned}
$$

which implies $d_{\{1, \ldots, L\}} \geq \bar{d}_{\{1, \ldots, L\}}$. Here

$$
\bar{d}_{\{1, \ldots, L\}}=\left(\sum_{i=1}^{L} \frac{1}{d_{\{i\}}}-\frac{L-1}{\sigma_{X}^{2}}\right)^{-1} \text {. }
$$

Conversely, if $d_{\{1, \ldots, L\}} \geq \bar{d}_{\{1, \ldots, L\}}$, then we must have $d_{\{1, \ldots, i\}} \geq\left(\frac{1}{d_{\{1, \ldots, i-1\}}}+\frac{1}{d_{\{i\}}}-\frac{1}{\sigma_{X}^{2}}\right)^{-1}$ for some $i \geq 2$, which implies $\sigma_{i-1}^{2}=0$ and further implies $\sigma_{1}^{2}=\cdots=$ $\sigma_{L-1}^{2}=0$

2) Case 2: $0<\sigma_{1}^{2} \leq \cdots \leq \sigma_{L}^{2}<\sigma_{X}^{2}$. The saddle point is uniquely determined by

$$
\begin{aligned}
& \left(\frac{\sigma_{X}^{2} d_{\{1, \ldots, i\}}}{\sigma_{X}^{2}-d_{\{1, \ldots, i\}}}+\sigma_{i-1}^{2}\right)^{-1} \\
& =\left(\frac{\sigma_{X}^{2} d_{\{1, \ldots, i-1\}}}{\sigma_{X}^{2}-d_{\{1, \ldots, i-1\}}}+\sigma_{i-1}^{2}\right)^{-1} \\
& \quad+\left(\frac{\sigma_{X}^{2} d_{\{i\}}}{\sigma_{X}^{2}-d_{\{i\}}}+\sigma_{i-1}^{2}\right)^{-1}, \quad i=2, \ldots, L \\
& \sigma_{i-1}^{2}=\sigma_{i}^{2} \text { if } \alpha_{i}=\alpha_{i+1}, \quad i=2, \ldots, L-1 \\
& d_{\{1, \ldots, i\}}=\frac{\left(\alpha_{i}-\alpha_{i+1}\right) \sigma_{X}^{2} \sigma_{i-1}^{2} \sigma_{i}^{2}}{\alpha_{i+1} \sigma_{i}^{2}\left(\sigma_{X}^{2}-\sigma_{i-1}^{2}\right)-\alpha_{i} \sigma_{i-1}^{2}\left(\sigma_{X}^{2}-\sigma_{i}^{2}\right)} \\
& \text { if } \alpha_{i}>\alpha_{i+1}, \quad i=2, \ldots, L-1 .
\end{aligned}
$$

3) Case 3: $0<\sigma_{1}^{2} \leq \cdots \leq \sigma_{i^{*}-1}^{2}<\sigma_{i^{*}}^{2}=\cdots=\sigma_{L-1}^{2}=$ $\sigma_{X}^{2}$, where $i^{*}=-1+\min \left\{i: \alpha_{i}=\alpha_{L}\right\}$ if $\alpha_{1}>\alpha_{L}$ and $i^{*}=1$ if $\alpha_{1}=\alpha_{L}$. Now $\sigma_{i}^{2}, i=1, \ldots, L-1$, and $d_{\{1, \ldots, i\}}, i=2, \ldots, i^{*}$, are uniquely determined by

$$
\begin{aligned}
& \left(\frac{\sigma_{X}^{2} d_{\{1, \ldots, i\}}}{\sigma_{X}^{2}-d_{\{1, \ldots, i\}}}+\sigma_{i-1}^{2}\right)^{-1} \\
& =\left(\frac{\sigma_{X}^{2} d_{\{1, \ldots, i-1\}}}{\sigma_{X}^{2}-d_{\{1, \ldots, i-1\}}}+\sigma_{i-1}^{2}\right)^{-1} \\
& \quad+\left(\frac{\sigma_{X}^{2} d_{\{i\}}}{\sigma_{X}^{2}-d_{\{i\}}}+\sigma_{i-1}^{2}\right)^{-1}, \quad i=2, \ldots, i^{*} \\
& \sigma_{i-1}^{2}=\sigma_{i}^{2} \text { if } \alpha_{i}=\alpha_{i+1}, \quad i=2, \ldots, i^{*}-1 \\
& \sigma_{i}^{2}=\sigma_{X}^{2}, \quad i=i^{*}, \ldots, L-1 \\
& d_{\{1, \ldots, i\} \quad\left(\alpha_{i}-\alpha_{i+1}\right) \sigma_{X}^{2} \sigma_{i-1}^{2} \sigma_{i}^{2}} \quad \text { if } \alpha_{i}>\alpha_{i+1}, \quad i=2, \ldots, i^{*}
\end{aligned}
$$


while $d_{\{1, \ldots, i\}}, i=i^{*}+1, \ldots, L-1$, must satisfy

$d_{\{1, \ldots, i\}} \geq d_{\{1, \ldots, i+1\}}-d_{\{i+1\}}+\sigma_{X}^{2}, \quad i=i^{*}, \ldots, L-1$.

Note that $d_{\{1, \ldots, L\}}$ does not appear in (43), (44), (45), and (46). Therefore, given $d_{\{i\}}, i=1, \ldots, L$, we can increase $d_{\{1, \ldots, L\}}$ without affecting $\sigma_{i}^{2}, i=1, \ldots, L-1$, and $d_{\{1, \ldots, i\}}, i=2, \ldots, i^{*}$, until

$$
d_{\{1, \ldots, L\}}=d_{\left\{1, \ldots, i^{*}\right\}}+\sum_{i=i^{*}+1}^{L} d_{\{i\}}-\left(L-i^{*}\right) \sigma_{X}^{2} .
$$

Denote this critical $d_{\{1, \ldots, L\}}$ by $\underline{d}_{\{1, \ldots, L\}}$. If we further increase $d_{\{1, \ldots, L\}}$, then the $d_{\left\{1, \ldots, i^{*}\right\}}$ induced by (43), (44), (45), and (46) cannot satisfy (47) anymore, and we enter the regime covered by case 2 . It is worth noting that

$$
\underline{d}_{\{1, \ldots, L\}}=\sum_{i=1}^{L} d_{\{i\}}-(L-1) \sigma_{X}^{2}
$$

when $\alpha_{2}=\cdots=\alpha_{L}$.

\section{REFERENCES}

[1] A. A. El Gamal and T. M. Cover, "Achievable rates for multiple descriptions," IEEE Trans. Inf. Theory, vol. IT-28, pp. 851-857, Nov. 1982.

[2] R. Venkataramani, G. Kramer, and V. K. Goyal, "Multiple description coding with many channels," IEEE Trans. Inf. Theory, vol. 49, pp. 2106-2114, Sep. 2003.

[3] S. S. Pradhan, R. Puri, and K. Ramchandran, " $n$-channel symmetric multiple descriptions-Part I: $(n, k)$ source-channel erasure codes," IEEE Trans. Inf. Theory, vol. 50, pp. 47-61, Jan. 2004.

[4] R. Puri, S. S. Pradhan, and K. Ramchandran, " $n$-channel symmetric multiple descriptions-Part II: An achievable rate-distortion region," IEEE Trans. Inf. Theory, vol. 51, pp. 1377-1392, Apr. 2005.

[5] C. Tian and J. Chen, "New coding schemes for the symmetric $K$-description problem," IEEE Trans. Inf. Theory, submitted for publication.
[6] L. Ozarow, "On a source coding problem with two channels and three receivers," Bell Syst. Tech. J., vol. 59, no. 10, pp. 1909-1921, Dec. 1980.

[7] H. Wang and P. Viswanath, "Vector Gaussian multiple description with individual and central receivers," IEEE Trans. Inf. Theory, vol. 53, pp. 2133-2153, Jun. 2007.

[8] J. Chen, C. Tian, T. Berger, and S. S. Hemami, "Multiple description quantization via Gram-Schmidt orthogonalization," IEEE Trans. Inf. Theory, vol. 52, pp. 5197-5217, Dec. 2006.

[9] X. Zhang, J. Chen, S. B. Wicker, and T. Berger, "Successive coding in multiuser information theory," IEEE Trans. Inf. Theory, vol. 53, pp. 2246-2254, Jun. 2007.

[10] M. H. M. Costa, "A new entropy power inequality," IEEE Trans. Inf. Theory, vol. IT-31, pp. 751-760, Nov. 1985.

[11] J. Edmonds, "Submodular functions, matroids and certain polyhedra," in Combinatorial Structures and Their Applications, R. Guy, $\mathrm{H}$ Hanani, N. Sauer, and J. Schonheim, Eds. New York: Gordon and Breach, 1970, pp. 69-87.

[12] C. Tian, S. Mohajer, and S. Diggavi, "Approximating the Gaussian multiple description rate region under symmetric distortion constraints," IEEE Trans. Inf. Theory, vol. 55, no. 6, pp. 3869-3891, Aug. 2009.

[13] S. N. Diggavi and T. M. Cover, "The worst additive noise under a covariance constraint," IEEE Trans. Inf. Theory, vol. 47, pp. 3072-3081, Nov. 2001

[14] R. Liu, T. Liu, H. V. Poor, and S. Shamai (Shitz), "A vector generalization of Costa's entropy-power inequality with applications," IEEE Trans. Inf. Theory [Online]. Available: http://arxiv.org/abs/0903.3024, submitted for publication

Jun Chen (S'03-M'06) received the B.E. degree with honors in communication engineering from Shanghai Jiao Tong University, Shanghai, China, in 2001, and the M.S. and Ph.D. degrees in electrical and computer engineering from Cornell University, Ithaca, NY, in 2003 and 2006, respectively.

He was a Postdoctoral Research Associate with the Coordinated Science Laboratory, University of Illinois at Urbana-Champaign, Urbana, from 2005 to 2006, and a Josef Raviv Memorial Postdoctoral Fellow with the IBM Thomas J. Watson Research Center, Yorktown Heights, NY, from 2006 to 2007. He is currently an Assistant Professor of Electrical and Computer Engineering with McMaster University, Hamilton, ON, Canada. He holds the Barber-Gennum Chair in Information Technology. His research interests include information theory, wireless communications, and signal processing. 